\title{
Suppression of the RAS/ERK pathway by Tmsb10 is the key step for promoting fetal Leydig cell differentiation from progenitors
}

\section{Miki Inoue}

Kyushu University https://orcid.org/0000-0003-3581-9852

\section{Takashi Baba}

Kyushu University

\section{Fumiya Takahashi}

Kyushu University

\section{Miho Terao}

National Research Institute for Child Health and Development

\section{Shogo Yanai}

Kyushu University

\section{Yuichi Shima}

Kyushu University

\section{Daisuke Saito}

Kyushu University

Kei Sugihara

Kyushu University

\section{Takashi Miura}

Kyushu University

\section{Shuji Takada}

National Research Institute for Child Health and Development

\section{Mikita Suyama}

Kyushu University https://orcid.org/0000-0001-9526-3193

\section{Yasuyuki Ohkawa}

Kyushu University https://orcid.org/0000-0001-6440-9954

Ken-ichirou Morohashi ( $\square$ morohashi.ken-ichirou.874@m.kyushu-u.ac.jp)

Kyushu University

\section{Article}

Keywords: 
Posted Date: February 16th, 2022

DOI: https://doi.org/10.21203/rs.3.rs-1357916/v1

License: (c) (1) This work is licensed under a Creative Commons Attribution 4.0 International License. Read Full License

Version of Record: A version of this preprint was published at Communications Biology on September 15th, 2022. See the published version at https://doi.org/10.1038/s42003-022-03941-5. 


\section{Suppression of the RAS/ERK pathway by Tmsb10 is the key step for promoting fetal}

\section{Leydig cell differentiation from progenitors}

3

4 Miki Inoue ${ }^{1,2}$, Takashi Baba ${ }^{1,2}$, Fumiya Takahashi ${ }^{1}$, Miho Terao ${ }^{3}$, Shogo Yanai ${ }^{1}$, Yuichi

5 Shima $^{1,2}$, Daisuke Saito ${ }^{1,4}$, Kei Sugihara ${ }^{5}$, Takashi Miura ${ }^{5}$, Shuji Takada ${ }^{3}$, Mikita Suyama ${ }^{1,4}$,

6 Yasuyuki Ohkawa ${ }^{1,5}$, Ken-ichirou Morohashi ${ }^{1,2 *}$

7

8 1, Department of Systems Life Sciences, Graduate School of Systems Life Sciences, Kyushu

9 University, Maidashi 3-1-1, Higashi-ku, Fukuoka, 812-8582, Japan.

10 2, Department of Molecular Biology, Graduate School of Medical Sciences, Kyushu

11 University, Maidashi 3-1-1, Higashi-ku, Fukuoka, 812-8582, Japan.

12 3, Department of Systems BioMedicine, National Research Institute for Child Health and

13 Development, 2-10-1 Okura, Setagaya-ku, Tokyo 157-8535, Japan.

14 4, Division of Bioinformatics, Medical Institute of Bioregulation, Kyushu University,

15 Maidashi 3-1-1, Higashi-ku, Fukuoka 812-8582, Japan.

16 5, Department of Anatomy and Cell Biology, Graduate School of Medical Sciences, Kyushu

17 University, Maidashi 3-1-1, Higashi-ku, Fukuoka, 812-8582, Japan.

18

$19 *$ To whom correspondence should be addressed. 
20 Department of Molecular Biology, Graduate School of Medical Sciences, Kyushu University,

21 Maidashi 3-1-1, Higashi-ku, Fukuoka 812-8582, Japan

22 e-mail; morohashi.ken-ichirou.874@m.kyushu-u.ac.jp

23

24 Disclosure statement: The authors have nothing to disclose. 


\section{ABSTRACT}

26 Leydig cells in fetal testes play crucial roles in masculinizing fetuses through androgen

27 production. Gene knockout studies have revealed that growth factors are implicated in fetal

28 Leydig cell (FLC) differentiation, but little is known about the mechanisms regulating this

29 process. We investigated this issue by characterizing FLC progenitor cells using single-cell

30 RNA sequencing. The sequence datasets suggested that thymosin $\beta 10$ (Tmsb10) was transiently

31 upregulated in the progenitors. While studying the function of Tmsb10, we revealed that

32 platelet-derived growth factor (PDGF) regulated ciliogenesis through the RAS/ERK and

33 PI3K/AKT pathways, and thereby promoted desert hedgehog (DHH)-dependent FLC

34 differentiation. Tmsb10 expressed in the progenitor cells induced their differentiation into FLCs

35 by suppressing the RAS/ERK pathway. Through characterizing the transiently expressed

36 Tmsb10 in the FLC progenitors, this study unveiled the molecular process of FLC

37 differentiation and showed that it is cooperatively induced by DHH and PDGF. 


\section{INTRODUCTION}

Two types of somatic cells, Sertoli and Leydig cells, play unique and mutually

complementary roles to achieve testicular functions. Sertoli cells provide germ cells with

41 nutrients and stimuli to support their differentiation, while Leydig cells produce a potent

42 androgen, testosterone, that regulates the differentiation and functions of both germ and Sertoli

43 cells ${ }^{1}$. In addition to these intratesticular functions, testosterone induces a variety of male traits

44 throughout the body. Unlike other vertebrates, mammalian species possess two developmentally

45 different Leydig cells, fetal-type Leydig cells (FLCs) and adult-type Leydig cells (ALCs), the

46 former of which play pivotal roles in the masculinization of fetuses through androgen

47 production $^{2}$.

48 Several studies using gene-disrupted mice have revealed the involvement of multiple

49 growth factors and their receptors in gonad development. Among them, desert hedgehog

50 (DHH), NOTCH, platelet-derived growth factor (PDGF), and transforming growth factor $\beta$

51 (TGF $\beta$ ) signals were implicated in FLC differentiation; disruption of these genes resulted in

52 aberrant FLC differentiation ${ }^{3-6}$. Likewise, genes encoding transcription factors such as aristaless

53 related homeobox $(A r x)^{7}$, podocyte-expressed 1/transcription factor $21(\text { Pod1/Tcf } 21)^{8}$, adrenal

54 4-binding protein/ steroidogenic factor-1 (Ad4BP/SF-1, Nr5al) $)^{9,10}$, glioma-associated Krüppel-

55 type $\mathrm{Zn}$ finger protein $($ Gli $1 / G l i 2){ }^{11}$, and $G l i 3^{12}$ were found to contribute to FLC differentiation

56 as demonstrated in gene knockout (KO) mice. Moreover, possible FLC progenitor cells have

57 been shown to express ARX ${ }^{13}, \mathrm{MAFB}^{14}, \operatorname{Notch}^{15,16}, \mathrm{Nestin}^{17}$, and $W n t 5 a^{18,19}$. Therefore, several 
58 studies have shown that multiple factors are involved in the differentiation of FLCs. However, it

59 remains unclear how these factors result in the formation of FLC progenitor cells and then

60 promote their successive differentiation into FLCs.

61 Studies concerning hedgehog $(\mathrm{HH})$ signaling have unveiled the complex mechanism of

62 intracellular signal transduction. Upon binding of $\mathrm{HH}$ to its receptor Patched (PTCH),

63 Smoothened (SMO) is released from inhibition by the receptor and then accumulates in the

64 primary cilium ${ }^{20}$. Thereafter, SMO undergoes phosphorylation, and in this form it promotes

65 dissociation of GLI from kinesin family protein 7 and Suppressor of fused. Ultimately, GLI is

66 converted to an active form and then begins to transcribe $\mathrm{HH}$ target genes ${ }^{21}$.

67 Studies of PDGF signaling have demonstrated that two receptors, PDGFR $\alpha$ and PDGFR $\beta$,

68 transduce signals upon binding to four ligand molecules, PDGF-A, PDGF-B, PDGF-C, and

69 PDGF-D ${ }^{22}$. The ligand-bound receptors activate the RAS/ERK pathway by successive

70 phosphorylation of its components. It has also been shown that RAS activates the PI3K/AKT

71 pathway, in which phosphatidylinositol 3-phosphate $\left(\mathrm{PIP}_{3}\right)$, which is synthesized by $\mathrm{PI} 3 \mathrm{~K}$, plays

72 a pivotal role in activating PDK1 and AKT. By regulating these signal pathways, PDGFs are

73 involved in a variety of cellular processes such as differentiation, proliferation, metabolism, and

74 migration $^{22}$. As described above, the specific mechanisms of DHH and PDGF signal

75 transduction have been uncovered gradually. Unfortunately, however, it remains largely

76 unknown how these growth factors promote FLC differentiation. 
$79 \beta$-thymosin family, have highly homologous amino acid sequences. The expression of these $\beta$ -

80 thymosins has been observed in a variety of normal and cancer cells $\mathrm{s}^{24-26}$. As for their functions,

81 TMSB4X was shown to sequester actin monomer to suppress the formation of filamentous

$82 \operatorname{actin}^{27}$. Likewise, TMSB10 suppresses actin polymerization through the actin-binding sequence

83 conserved in these $\beta$-thymosins ${ }^{28}$. Related to their actin-sequestering function, many studies

84 have reported that these $\beta$-thymosins are potentially involved in processes such as blood vessel

85 formation, wound healing, cell migration, and cancer metastasis ${ }^{29}$. Moreover, TMSB10, but not

86 TMSB4X, interacts directly with RAS to inhibit RAS-RAF interaction, which disturbs

87 downstream signal transduction ${ }^{30}$.

88 In the present study, we examined the interstitial cells of developing fetal testes in mice

89 using single-cell RNA sequencing (scRNA-seq). Analyses of the sequence datasets found a

90 unique cell fraction potentially consisting of FLC progenitors. Among the genes whose

91 expression was upregulated in the progenitor cells, we focused on Tmsb10. We found that

92 PDGF regulated the formation of primary cilia via signaling in the RAS/ERK and PI3K/AKT

93 pathways, and moreover that TMSB10 promoted ciliation by hindering the interaction between

94 RAS and RAF, thereby suppressing the RAS/ERK signal pathway. This study unveils, for the

95 first time, part of the molecular process of FLC differentiation that is induced cooperatively by

96 DHH and PDGF. 


\section{FLC progenitor cells are present in the interstitial space of fetal testes}

99

100

101

102

103

104

105

106

107

108

109

110

111

112

A transgenic FLE-EGFP mouse was established using the fetal Leydig-specific enhancer (FLE) and promoter region of $A d 4 B P / S F-1$ gene ${ }^{31}$. FLCs in the fetal testes of the transgenic mice were strongly labeled with EGFP (S-EGFP cells), and a large population of interstitial cells was labeled only weakly with EGFP (W-EGFP cells). Both S-EGFP and W-EGFP cells could be recovered separately by fluorescence-activated cell sorting (FACS) from E16.5 fetal testes $^{32}$. Because FLCs at this stage increase in number even though they scarcely proliferate ${ }^{13,17,33}$, we anticipated that the W-EGFP cell population may include FLC progenitor cells. Indeed, in vitro testis reconstruction studies demonstrated that some W-EGFP cells, if not all, have the potential to differentiate into $\mathrm{FLCs}^{34}$.

To identify the FLC progenitors, 696 W-EGFP cells and 92 S-EGFP cells prepared from E16.5 fetal testes were subjected to scRNA-seq ${ }^{35}$. After low-quality scRNA-seq datasets were removed, 341 and 80 datasets obtained from the W-EGFP and S-EGFP cells, respectively, were subjected to subsequent analyses (Supplementary Table 1).

To assess how many cell types were present among the W-EGFP cells, the datasets were subjected to hierarchical clustering on the significant principal components. As indicated in the cluster dendrogram in Fig. 1a, the W-EGFP cells were divided into three clusters (clusters A, B, and C), while the S-EGFP cells were divided into two clusters (clusters D and E). According to the relative distances between the clusters, cluster $\mathrm{E}$ was the most distant from the other 
117 clusters, while clusters A and B were not clearly segregated. Interestingly, clusters C and D,

118 which were originally derived from the W-EGFP and S-EGFP cells, respectively, demonstrated

119 an intimate correlation. A similar distribution of the five cell clusters was observed by t-

120 distributed stochastic neighboring embedding (t-SNE) (Fig. 1b).

121 The scRNA-seq data were further subjected to Monocle trajectory analysis to predict the

122 developmental trajectory of the five cell clusters. As indicated in Fig. 1c, the cells in clusters A

123 and B were predicted to differentiate into those in cluster C, and eventually, via cluster D, into

124 those in cluster E. Because the cells in cluster E are FLCs, as described later, characterization of

125 the putative progenitor cells in cluster $\mathrm{C}$ seemed to be critical for uncovering the mechanism of

126 FLC differentiation.

127 Therefore, a heatmap of gene expression was generated using the genes whose expression

128 was altered in clusters C, D, and E (Fig. 1a). The genes in group I showed higher expression in

129 cluster E and/or cluster D. FLC marker genes such as insulin-like 3 (Insl3 $)^{36,37}$, cholesterol side-

130 chain cleavage enzyme cytochrome 450 (Cypl 1al), and 3 $\beta$-hydroxysteroid dehydrogenase

$131(H s d 3 b 1)^{38-40}$ were included in this group, indicating that the cells in clusters D and E consisted

132 of immature and mature FLCs, respectively. The genes in group II showed higher expression in

133 clusters C, D, and E. Interestingly, several genes encoding large ribosomal subunits were

134 included in this group. Finally, the genes in group III showed higher expression in cluster C.

136 Expression of Tmsb10 is transiently upregulated in putative FLC progenitors 
137 Expression levels of the genes above were depicted colorimetrically on the cells whose

138 distribution was determined by t-SNE (Fig. 1d-f, Supplemental Fig 1a). As expected, the

139 highest expression of Cypl Ial was seen in cluster E, that of calreticulin (Calr) was in clusters

$140 \mathrm{C}, \mathrm{D}$, and E, and that of Tmsb10 was in cluster C. Considering that the cells in cluster C are

141 FLC progenitors, the expression of Tmsb10 appeared to be transiently upregulated during

142 differentiation into FLCs. The expression pattern of Tms $b 4 x$, another member of $\beta$-thymosin

143 family, was different from that of Tmsb10 (Supplementary Fig. 1b). We previously showed that

144 the expression of ARX is gradually decreased in interstitial cells that are differentiating into

145 FLCs $^{13}$. Consistent with the previous observation, Arx expression appeared to be decreased in

146 cluster $\mathrm{C}$ and then further decreased in clusters D and E (Fig. 1g).

148 TMSB10 is expressed in the interstitial cells of fetal testes

149 Focusing on the gene expression profile of the genes in group III, we investigated whether

$150 T m s b 10$ is involved in FLC differentiation. First, we attempted to identify the cells expressing

151 TMSB10 in fetal testes. Because no available antibody recognized TMSB10, we generated

152 knock-in mice in which $m$ Cherry was inserted at the Tmsb10 locus (Supplementary Fig. 2). The

153 mice were then crossed with FLE-EGFP mice, and then the fetal testes of the double transgenic

154 mice at E16.5 were examined. TMSB10 (mCherry) did not seem to be expressed in FLCs (S-

155 EGFP cells) (open arrowheads in Fig. 2a). Strong signals for TMSB10 were detected in the

156 interstitial space, and they were colocalized with laminin (arrows). Considering that laminin is a 
157 marker of endothelial cells (Supplementary Fig. 3), TMSB10 was thought to be strongly

158 expressed in the cells. Further, cells exhibiting weak TMSB10 signals were present in the

159 interstitial space (closed arrowheads), and some of these cells were weakly stained with EGFP.

160 To exclude the possibility that the W-EGFP cell population includes Tmsb10-expressing

161 endothelial cells, we examined the expression of the endothelial cell marker Pecam 1 in W-

162 EGFP, S-EGFP, and EGFP-negative cells. qRT-PCR revealed that Pecam1-positive endothelial

163 cells were mostly recovered in EGFP-negative cells but not in W-EGFP cells (Fig. 2b-d),

164 indicating that W-EGFP cells include TMSB10-positive cells other than endothelial cells.

Tmsb10 is required for FLC differentiation

167

As described previously ${ }^{34}$, we established a testis reconstruction system using W-EGFP cells mixed with whole cells prepared from wild-type fetal testes (Fig. 3a). Using this system, we succeeded in recapitulating FLC differentiation from W-EGFP cells by detecting the appearance of cells strongly positive for EGFP (equivalent to S-EGFP cells) (Fig. 3b). A

171 previous gene disruption study demonstrated that DHH is required for differentiation of FLCs ${ }^{3}$.

172 Thus, we investigated whether DHH stimulates FLC differentiation using in vitro reconstructed

173 testes. As shown in Fig. 3c and d, EGFP signals in the reconstructed testes increased when

174 incubated in the presence of a SMO agonist (SAG).

175 Considering the transiently upregulated expression of $T m s b 10$ in the putative progenitor

176 cells, we hypothesized that Tmsb10 plays a critical role in FLC differentiation. Thus, we 
177 examined whether Tmsb10 knockdown (KD) impacted FLC differentiation in the reconstructed

178 testes. Cultured W-EGFP cells were treated with the siRNAs for Tmsb10, Tmsb4x, and

$179 A d 4 B P / S F-1$. As expected, these treatments resulted in a clear reduction of the expression of

180 each corresponding gene (Supplementary Fig. 4). Then, we utilized these KD cells for in vitro

181 testis reconstruction assays. Tmsb10 KD was found to impair the ability of W-EGFP cells to

182 differentiate into S-EGFP FLCs, although this impairment was not observed following

183 treatment with siTmsb4x or control siRNA (siCnt) (Fig. 3e). It has been established that

$184 A d 4 B P / S F-1$ is essential for steroidogenic cell differentiation ${ }^{9,10,41,42}$. As expected, FLC

185 differentiation was markedly affected by $A d 4 B P / S F-1$ KD (Fig. 3e). Quantitative examination

186 indicated that Tmsb10 KD decreased the differentiation efficacy to $37 \%$ of control, whereas it

187 was unaffected by $T m s b 4 x \mathrm{KD}$ (Fig. 3f). $A d 4 B P / S F-1 \mathrm{KD}$ resulted in a reduction of more than

$18890 \%$. Similar effects of Tmsb10 KD on FLC differentiation were observed in the presence of

189 SAG (Fig. 3g, h).

190 Finally, we examined whether activity downstream of $\mathrm{HH}$ signaling was affected by

191 Tmsb10 KD. Gli1 gene expression is known to be activated by HH signaling ${ }^{21}$. As expected,

192 Tmsb10 KD resulted in a decrease of Glil expression (Fig. 3i), while Tmsb4x KD had no effect

193 (Supplementary Fig. 5a-c). Taken together, these results strongly suggest that Tmsb10 is

194 required for FLC differentiation through regulating HH signaling.

195

196 Tmsb10 regulates primary cilia formation by inhibiting the RAS/ERK pathway 
198 transduction ${ }^{20}$, we examined whether $T m s b 10$ was implicated in the regulation of ciliogenesis.

199 Consistent with the observation that primary cilia emerge only during the G0/G1 cell cycle

200 phase $^{43}$, we scarcely detected them in W-EGFP cells cultured in serum-containing medium.

201 Therefore, W-EGFP cells were cultured in serum-free medium for $24 \mathrm{~h}$ before the following

202 immunofluorescence studies were performed. Under this condition, approximately $70 \%$ of the

203 W-EGFP cells were ciliated. Notably, the number of ciliated cells was dose-dependently

204 decreased by siTmsb10 treatment (Fig. 4a, b), whereas siCnt treatment had no effect. The

205 number of ciliated cells was decreased to $45 \%$ relative to baseline by KD with siTmsb10 at a

206 concentration of $10.0 \mathrm{nM}$. Treatments with higher concentrations resulted in decreased survival

207 of W-EGFP cells. Therefore, the following Tmsb10 KD experiments were performed using a

208 concentration of $10.0 \mathrm{nM}$. A similar decrease in the number of ciliated cells was seen when W-

209 EGFP cells cultured in the presence of SAG were subjected to Tmsb10 KD (Supplementary Fig.

210 6). By contrast, Tmsb4x KD had no effect on ciliation (Supplementary Fig. 5d). Next, we

211 examined whether ciliation and FLC differentiation were simultaneously suppressed by

212 siTmsb10 in a dose-dependent manner. Testes were reconstructed with W-EGFP cells treated

213 with increasing concentrations of siTmsb10. As shown in Fig. 4c, FLC differentiation was

214 suppressed dose dependently by siTmsb10. Likewise, siTmsb10 dose-dependently decreased

215 Gli1 gene expression in cultured W-EGDFP cells (Fig. 4d). 
We next attempted to determine the mechanism whereby $T m s b 10$ regulates ciliogenesis.

217 Regarding the function of TMSB10, experiments using human cancer cell lines showed that the

218 protein binds directly to RAS, and thereby suppresses RAS-RAF interaction ${ }^{30}$. Moreover, this

219 suppression ultimately resulted in failure of ERK phosphorylation and activation. Based on

220 these findings, we planned to confirm the interaction between TMSB10 and RAS in W-EGFP

221 cells with pull-down assays using ectopically expressed Flag-tagged TMSB10. Unfortunately,

222 however, we could not find an efficient method for plasmid DNA transfection into W-EGFP

223 cells. Thus, the interaction was confirmed in HEK293 cells (Fig. 4e). Subsequently, ERK

224 phosphorylation, an event that occurs downstream of RAS/RAF activation, was examined in

225 siCnt- or siTmsb10-treated W-EGFP cells. The amount of phosphorylated ERK (pERK) was

226 increased by Tmsb10 KD both in the presence and absence of SAG, whereas the amount of

227 ERK was unaffected (Fig. 4f, g).

228 Taken together, these results indicated that TMSB10 promotes ciliogenesis and suppresses

229 the RAS/ERK pathway. However, it remained unclear whether these two effects were

230 connected. Several prior studies demonstrated that ciliogenesis was suppressed by the

231 RAS/ERK pathway ${ }^{44-46}$. Therefore, as shown in Fig. 4h, we tentatively assumed that TMSB10

232 promotes ciliogenesis by suppressing the RAS/ERK pathway. To test this assumption, we

233 examined the functional correlation between Tmsb10 and Ras during FLC differentiation. W-

234 EGFP cells treated with siTmsb10 and/or siRas were used for testis reconstruction assays.

235 Similar to the results shown in Fig. 3g and h, FLC differentiation was decreased by Tmsb10 KD 
236 (Fig. 4i). Consistent with the aforementioned assumption (Fig. 4h), FLC differentiation was

237 increased by Ras KD. Moreover, the decrease in FLC differentiation by Tmsb10 KD was

238 canceled by simultaneous KD of Ras. The expression of the Gli1 gene in cultured W-EGFP

239 cells was similarly affected by treatment with these siRNAs (Fig. 4j).

240 We next examined the effect of Ras KD on ciliogenesis. Again, Tmsb10 KD decreased the

241 number of ciliated cells to $43 \%$ relative to control (Fig. $4 \mathrm{k}$ ). Considering the effects of the KDs

242 described above, we expected that Ras KD would increase the number of ciliated cells.

243 Unexpectedly, however, this was not the case. As noted above, at most about 70\% of W-EGFP

244 cells were ciliated even when they were cultured in serum-free medium, suggesting that this was

245 the maximum percentage that could be ciliated under this condition. This assumption seemed to

246 be supported by a double-KD study with siTmsb10 and siRas. Although KD of only Ras had no

247 effect, the decrease of ciliated cells by $T m s b 10 \mathrm{KD}$ was mitigated by Ras KD, and their

248 numbers reached those seen following treatment with siCnt.

$250 \quad$ PDGF possibly regulates ciliogenesis

251 As indicated in Fig. 4h, the RAS/ERK pathway seemed to be involved in FLC

252 differentiation. However, it remained unclear which molecule(s) activated RAS in W-EGFP

253 cells. We considered PDGF to be a possible candidate because FLC differentiation was largely

254 abrogated by Pdgfra gene disruption ${ }^{4}$, and PDGF signaling activated the RAS/ERK pathway ${ }^{47}$. 

testes. As shown in Fig. 5a, FLC differentiation was not activated by PDGF-AA alone.

257 Interestingly, however, PDGF-AA further enhanced the differentiation induced by SAG

258 treatment. Likewise, Gli1 gene expression was increased by PDGF-AA in the presence but not 259 the absence of SAG (Fig. 5b).

260 Next, we examined whether PDGF affected ERK phosphorylation in W-EGFP cells.

261 Consistent with previous studies ${ }^{47}$, PDGF-AA increased the amount of pERK, although the

262 amount of ERK was unchanged (Fig. 5c, d). SAG treatment had no effect on the amount of

263 pERK. These results demonstrated that PDGF-AA increased pERK, probably by activating

264 RAS. Since TMSB10 was shown to suppress the RAS/ERK pathway (Fig. 4f), it was expected

265 that PDGF-promoted ERK phosphorylation would be increased by $T m s b 10 \mathrm{KD}$, and this was

266 found to be the case (Fig. 5e, f). Based on these observations, it is likely that PDGF, in the

267 presence of HH signaling, promotes FLC differentiation by activating the RAS/ERK pathway

268 (Fig. 5g).

269 However, we noticed the following inconsistency. As described above, the RAS/ERK

270 pathway was shown to suppress ciliogenesis ${ }^{44-46}$. In addition, the present study demonstrated

271 that PDGF activated the RAS/ERK pathway in W-EGFP cells. These observations suggest that

272 PDGF activates the RAS/ERK pathway and then suppresses ciliogenesis, resulting in

273 suppression of DHH-dependent FLC differentiation (Fig. 5g). Nevertheless, Fig. 5a shows that

274 PDGF promotes FLC differentiation cooperatively with DHH. This apparent inconsistency 
275 strongly suggests that another pathway functions downstream of the PDGF receptor to modulate

276 the suppression of ciliogenesis by the RAS/ERK pathway. In fact, ciliogenesis did not seem to

277 be markedly affected by PDGF-AA (Fig. 5h).

278

279 Role of the PDGF-activated PI3K/AKT pathway in FLC differentiation

280 It has been established that PDGF signaling activates the PI3K/AKT pathway, in which

$281 \mathrm{PIP}_{3}$, which is generated from $\mathrm{PIP}_{2}$ by PI3K, activates AKT (Fig. 6a) ${ }^{47,48}$. Thus, we investigated

282 the possibility that this pathway regulates FLC differentiation by activating ciliogenesis. First,

283 we examined whether AKT phosphorylation was promoted by PDGF-AA in W-EGFP cells. As

284 expected, the amount of phosphorylated AKT was increased in the presence of PDGF-AA,

285 whereas that of AKT was not affected (Fig. 6b, c). Next, we examined whether the PI3K/AKT

286 pathway regulates FLC differentiation in testis reconstruction assays using W-EGFP cells

287 treated with siRNA for Akt and phosphatase and tensin homolog (Pten). PTEN is known to

288 suppress the PI3K/AKT pathway by mediating the conversion of $\mathrm{PIP}_{3}$ to $\mathrm{PIP}_{2}\left(\right.$ Fig. 6a) ${ }^{48}$. When

289 the reconstructed testes were incubated in the presence of SAG and PDGF-AA, Akt KD

290 suppressed FLC differentiation. Conversely, Pten KD increased FLC differentiation (Fig. 6d).

291 Likewise, the expression of Gli1 in W-EGFP cells was decreased and increased by Akt KD and

292 Pten KD, respectively (Fig. 6e). The Tmsb10 KD-induced decreases in the FLC differentiation

293 rate and in Gli1 gene expression were observed in the presence of SAG and PDGF-AA. 
294 We next examined the possible role of the PI3K/AKT pathway in the regulation of

295 ciliogenesis. As shown in Fig. 6f, Akt KD decreased the number of ciliated W-EGFP cells in the

296 presence of SAG and PDGF-AA. Pten KD was expected to increase the number of ciliated

297 cells. However, as was the case with Ras KD (Fig. 4k), this increase was not observed.

298 Because several studies have demonstrated that activated AKT suppresses the RAS/ERK

299 pathway by reducing RAF activity ${ }^{49}$, we assumed that activating the PI3K/AKT pathway would

300 reduce ERK phosphorylation and eventually promote FLC differentiation through ciliogenesis.

301 Therefore, we examined whether inhibition of the PI3K/AKT pathway impacted ERK

302 phosphorylation. As expected, the PI3K inhibitor wortmannin decreased AKT phosphorylation

303 and increased ERK phosphorylation in W-EGFP cells (Fig. 6g, h). By contrast, when

304 PI3K/AKT signaling was activated by Pten KD, AKT phosphorylation and ERK

305 phosphorylation were increased and decreased, respectively (Fig. 6i, j). Together, these results

306 suggest that activation of the PI3K/AKT pathway promotes FLC differentiation by enhancing

307 ciliogenesis via suppression of the RAS/ERK pathway (Fig. 6a, Supplementary Fig. 7). 


\section{DISCUSSION}

309 FLCs are known to increase in number in the fetal testes until late pregnancy, even though

310 they rarely proliferate. Therefore, this increase has been thought to be due to the differentiation

311 of progenitor cells into FLCs ${ }^{13-19}$. The interstitial space of the developing fetal testes is occupied

312 predominantly by uncharacterized cells, in addition to smaller numbers of FLCs, peritubular

313 myoid cells, endothelial cells, and macrophages. According to studies published so far, FLC

314 progenitors are thought to be present in the interstitial space as cells positive for MAFB ${ }^{14}$,

$315 \mathrm{ARX}^{7,13}$, and/or Notch ${ }^{15,16}$ and Nestin ${ }^{17}$. In addition, our testis reconstruction study demonstrated

316 the presence of FLC progenitors among the uncharacterized interstitial cells (W-EGFP cells) ${ }^{34}$.

317 Based on these findings, it seemed critical to determine what kinds of cells constitute the

318 uncharacterized W-EGFP cell population.

$320 \quad$ Identification of FLC progenitor cells by scRNA-seq

321 scRNA-seq is a powerful technique for characterizing cells that contain one or more

322 unidentified cell populations. Indeed, novel cell populations have frequently been unveiled by

323 this technique ${ }^{50}$. So far, studies have obtained single-cell transcriptomes from the cells

324 constituting the fetal and postnatal testes ${ }^{18,51-55}$. These studies predicted the cellular lineages of

325 FLCs and ALCs, as well as those of germ and Sertoli cells. Eventually, it was hypothesized that

326 FLCs originate from uncharacterized interstitial cells. Unfortunately, however, none of the

327 studies identified a particular cell population as segregating and differentiating into FLCs. 
328 Although we currently do not know why these studies did not identify the cells that we found in

329 the present study, it might be because these single-cell studies utilized the overall population of

330 testicular cells, which included germ cells. Therefore, their contents demonstrate high biological

331 complexity, which might make it difficult to identify a population consisting of small number of

332 differentiating cells.

333 In contrast to these studies, Stevant et al. utilized EGFP-positive somatic cells from Nr5al-

334 BAC-EGFP transgenic mice ${ }^{18}$. The developmental lineages of these cells were examined using

335 several stages of the developing testes (E10.5 to E16.5). Eventually, the authors identified

336 multipotent progenitor cells that gave rise to Sertoli and Leydig cells. Moreover, a recent study

337 by the same group demonstrated that Wnt5a-expressing cells in E11.5 to E12.5 testes were

338 potentially progenitors of both FLCs and ALCs ${ }^{19}$.

339 Our current study utilized a simple cell population, namely W-EGFP cells, that probably

340 corresponds to uncharacterized interstitial cells. Ultimately, we identified FLC progenitor cells

341 characterized by the transient expression of Tmsb10. Utilization of a simple cell population may

342 have emphasized subtle differences and thereby enabled us to identify the small population of

$343 \quad$ FLC progenitors.

344 The developmental relation between progenitors characterized by Wnt5a versus Tmsb10

345 might be interesting. Considering that the expression of Wnt5a reaches a peak at E12.5-E13.5

346 and declines at E16.5 ${ }^{19}$, it might be reasonable to assume that the multipotent progenitor cells of

347 FLCs and ALCs are established at an early stage by the expression of $W n t 5 a$. Thereafter, FLC 
348 progenitors may be defined more selectively by the expression of Tmsb10. Because the cells

349 used in the two studies with $W n t 5 a$ and Tmsb10 were derived during distinct developmental

350 stages, the studies might successfully identify progenitor cells at the distinct stages. Further

351 investigations to determine the developmental relation between Tmsb10-positive and Wnt5a-

352 positive cells, and also between previously identified Mafb-positive, Arx-positive, and Notch-

353 and Nestin-positive cells might help us to clarify the entire process of FLCs differentiation.

Tmsb10 as a switch to promote FLC differentiation by suppressing the RAS/ERK pathway

It is known that both $T m s b 10$ and $T m s b 4 x$ possess actin sequestration activity, and thereby suppress actin polymerization ${ }^{27,28} . T M S B 10$ was found to be widely expressed in a variety of developing tissues, such as tooth germ ${ }^{56}$, antler growth center $^{57}$, post-implantation embryos ${ }^{25}$, ovarian follicles ${ }^{58}$, and brain ${ }^{59}$. In addition, elevated expressions of TMSB10 were observed in a variety of cancers $^{29}$. Because overexpression of TMSB10 resulted in the disappearance of Factin and thereby enhanced migration and invasion activities ${ }^{30}$, the functions of TMSB10 in cancers have been investigated based on the assumption that the protein acts as an actinmediated tumor suppressor.

364 In addition, an interesting study published in 2005 showed that TMSB10 hindered RAS-

365 RAF interaction, thereby suppressing the RAS/ERK signaling pathway as well as angiogenesis

366 and tumor growth ${ }^{30}$. Consistent with these findings, another study indicated that TMSB10

367 reduced cancer cell activities by suppressing the ERK pathway ${ }^{29}$. Together, these studies 
368 indicated that decreased expression of TMSB10 promotes tumor growth. In contrast, however,

369 increased expression of TMSB10 was associated with high malignant potential in cancers ${ }^{29}$.

370 Interestingly, a study of breast cancer revealed that enhanced expression of TMSB10 promoted

371 proliferation and migration of cancer cells by activating AKT/FOXO signaling ${ }^{60}$. Although the

372 functions of TMSB10 in cancer cells remain controversial, these findings regarding the

373 potential of TMSB10 to regulate signal pathways showed that it has functions beyond those

374 related to actin sequestration. In fact, our current study demonstrated that TMSB10 suppresses

375 the RAS/ERK pathway in W-EGFP cells. Moreover, we found for the first time that Tmsb10

376 promotes the differentiation of FLC progenitors.

377

378 Role of Tmsb10 in PDGF-DHH signal crosstalk through regulation of ciliation

379 PDGF plays crucial roles in the differentiation of various cell types by activating the

380 RAS/ERK and PI3K/AKT pathways ${ }^{47}$. As for the functional correlation between the two

381 pathways, it was found that the PI3K/AKT pathway may suppress the RAS/ERK pathway, and

382 thus these two pathways exert opposing effects on cell differentiation ${ }^{49}$. Likewise, our present

383 study demonstrated that PDGF can activate both the RAS/ERK and PI3K/AKT pathways,

384 which suppress and promote FLC differentiation, respectively. This bidirectional activity of

385 PDGF may maintain the balance between promotion and suppression of FLC differentiation,

386 and thereby sustain the ability of some W-EGFP cells, if not all, to differentiate into FLCs. 
388 However, it remains unclear whether these growth factors function cooperatively or

389 independently in the differentiation process. Regarding this issue, our testis reconstruction

390 assays fortunately showed that PDGF promotes FLC differentiation only in the presence of HH

391 signaling. This finding strongly suggested the presence of crosstalk between PDGF and DHH

392 signals. Eventually, we found that PDGF regulates the formation of cilia required for DHH

393 signal transduction, confirming that crosstalk occurs between the two growth factors.

394 A previous study demonstrated crosstalk between PDGF and TGF $\beta$ during osteogenic

395 differentiation ${ }^{61}$. Interestingly, the study showed that TGF $\beta$-induced osteogenic differentiation

396 was markedly enhanced by PDGF even though PDGF alone failed to promote the

397 differentiation. Another study revealed that lens differentiation was regulated by antagonistic

398 interaction between the PDGF-driven RAS/ERK pathway and the FGF-driven PI3K/AKT

399 pathway $^{62}$. In addition to the crosstalk between PDGF and both TGF $\beta$ and FGF, we revealed for

400 the first time the presence of crosstalk between PDGF and HH signaling.

401 Our current study investigated Tmsb10, which was identified by single-cell transcriptome

402 analyses of the uncharacterized interstitial cell population of the fetal testes, and provided

403 several fundamental clues regarding the mechanism underlying FLC differentiation. To obtain a

404 more comprehensive understanding of FLC differentiation, it might be critical to identify the

405 mechanism whereby Tmsb10 expression is selectively induced in a certain population of

406 interstitial cells and how it is transiently expressed prior to differentiation into FLCs. 
Preparation of EGFP cells with strong (S-EGFP) or weak (W-EGFP) staining

We previously established FLE-EGFP mice using FLE (fetal Leydig enhancer) of the $A d 4 B P / S F-1$ gene $^{31}$. EGFP-labeled transgenic testes were harvested at E16.5 and were dispersed

411 with collagenase (Sigma-Aldrich, St. Louis, MO, USA) and dispase (Thermo Fisher Scientific,

412 Waltham, MA, USA) as described previously ${ }^{32}$. Using JSAN (Bay bioscience, Kobe, Japan), the 413 dispersed cells were fractionated by FACS into N-EGFP, W-EGFP, S-EGFP populations based

414 on the negative, weak, or strong EGFP fluorescence intensity, respectively. All protocols for the 415 animal experiments were approved by the Animal Care and Use Committee of Kyushu

416 University, and all experiments were performed in accordance with the institutional guidelines.

$\operatorname{scRNA-seq}$

Single S-EGFP or W-EGFP cells were plated by FACS (SH800, Sony, Tokyo, Japan) into individual wells of a 384-plate (Piko PCR Plate, Thermo Fisher Scientific) pre-loaded with lysis

421 buffer. The CEL-Seq2 protocol established by Hashimshony et al. was used for RNA extraction 422 and library preparation ${ }^{35}$. Briefly, the RNA of each cell was reverse transcribed using CEL-Seq 423 primers containing an anchored poly(T), a 6-bp unique molecular identifier (UMI), a 5' Illumina 424 adapter (San Diego, CA, USA), a cell-specific 6-bp barcode, and a T7 promoter (Supplementary 425 Table 2). The External RNA Controls Consortium (ERCC) spike-ins (Thermo Fisher Scientific) 


\section{Data analysis of single-cell transcriptomes}

433 The quality check of the raw sequence reads was performed using FastQC (version 0.11.7),

434 and thereafter the reads were analyzed according to the CEL-Seq2 pipeline ${ }^{35}$. First, the reads of

435 CEL-Seq2 libraries were demultiplexed to each cell using the CEL-Seq barcodes. To identify

436 the transcript, the reads were mapped to the mouse reference genome (mm10) by Bowtie 2

437 software (version 2.3.4.1) ${ }^{63}$. PCR duplicates were removed using UMI information. The

438 mapped reads were counted using HTSeq (version 0.9 .1$)^{64}$. The quality of the sequence data

439 was further evaluated using a previously published method $^{65}$; low-quality samples were

440 removed by setting certain thresholds of low total reads, few expressed genes $(<1000$ genes $)$,

441 and high spike-in proportions.

442 Cells in the G1 cell cycle phase were selected to avoid potential confounding effects from

443 cell cycle-induced differences. Expression levels of the remaining genes were normalized and

444 denoised. These data were subjected to hierarchical clustering on the significant principal

445 components to divide cells into clusters. Differentially expressed genes (DEGs) of the clusters

446 were identified to characterize the clusters. R packages including SingleCellExperiment 
447 (version 1.0.0), scater (version 1.6.3), scran (version 1.6.9), Seurat (version 3.0.2), and Monocle

$448 \quad$ (version 2.6.4) were used.

449

450

siRNA treatments

451

W-EGFP cells were cultured on Advanced TC 24-well plates (Greiner Bio-One,

452 Kremsmünster, Austria) in $\alpha$-modified Eagle's medium ( $\alpha$-MEM, Nacalai Tesque, Kyoto,

453 Japan) supplemented with 10\% fetal bovine serum (FBS, Thermo Fisher Scientific) and

454 penicillin and streptomycin (PS, Thermo Fisher Scientific) at $37{ }^{\circ} \mathrm{C}$ under $5 \% \mathrm{CO}_{2}$ for $24 \mathrm{~h}$. The

455 cells were transfected with siRNAs using lipofectamine RNAiMAX reagent (Thermo Fisher

456 Scientific) for $24 \mathrm{~h}$. The siRNAs used in this study are listed in Supplementary Table 3. A

457 control siRNA (Stealth RNAi Negative Control Medium GC Duplex; Thermo Fisher Scientific)

458 was used as a negative control. The siRNA-treated cells were then utilized for qRT-PCR

459 analyses, in vitro testis reconstruction, immunocytochemistry, and Western blotting. To

460 investigate the effects of growth factor signals, the siRNA-treated cells were further cultured in

461 the presence of SAG (0.5 $\mu \mathrm{M}$; Adipogen Life Sciences, San Diego, CA, USA), mouse PDGF-

462 AA (10 ng/mL; Sigma-Aldrich), wortmannin (0.1 $\mu \mathrm{M}$; Cayman, Ann Arbor, MI, USA), or

463 dimethyl sulfoxide (DMSO, Nacalai Tesque) as a negative control.

464

465

Immunostaining 
467 were boiled for $5 \mathrm{~min}$ in $10 \mathrm{mM}$ sodium citrate ( $\mathrm{pH}$ 6.0) to unmask antigen epitopes ${ }^{34}$. The

468 sections were incubated with primary antibodies in blocking buffer ( $2 \%$ skim milk (WAKO,

469 Tokyo, Japan) in PBS) overnight at $4{ }^{\circ} \mathrm{C}$, and subsequently with secondary antibodies in the

470 blocking buffer for $1 \mathrm{~h}$ at room temperature. The primary and secondary antibodies used in this

471 study are listed in Supplementary Table 4. Nuclei were counterstained with DAPI (Sigma-

472 Aldrich).

473 To investigate ciliogenesis by immunofluorescence, W-EGFP cells cultured on $\mu$-Plate 96-

474 well TC $\left(2.0 \times 10^{4}\right.$ cells/well $)$ (ibiTreat, ibidi, Martinsried, Germany) in the same medium as

475 above for $24 \mathrm{~h}$ were treated with the siRNAs described above. After the siRNA treatment, the

476 cells were cultured in this medium again for $24 \mathrm{~h}$. Thereafter, they were fixed with $4 \%$

477 paraformaldehyde in PBS for $15 \mathrm{~min}$ at room temperature, and permeabilized with $0.5 \%$ Triton

$478 \mathrm{X}-100$ (WAKO) in PBS for 10 min followed by incubation in blocking buffer (1\% skim milk in

479 PBS) for $20 \mathrm{~min}$ at room temperature. Subsequently, the cells were treated with mouse anti-

480 ARL13B antibody overnight at $4{ }^{\circ} \mathrm{C}$, and then with Alexa Fluor 555-labeled goat anti-mouse

481 IgG antibody for $1 \mathrm{~h}$ at room temperature (Supplementary Table 4). Nuclei were counterstained 482 with DAPI.

483 Immunofluorescence images were captured using an LSM 700 confocal laser scanning

484 microscope (Carl Zeiss, Jena, Germany) and a BZ-X700 fluorescence microscope (Keyence, 
485 Osaka, Japan). The effects of the siRNA, SAG, and PDGF treatments on ciliogenesis were

486 evaluated as the percentage of ciliated cells in at least 500 overall cells in each sample $(n=5)$.

487

488

qRT-PCR analyses

489

qRT-PCR was performed as previously described ${ }^{66}$. In brief, total RNAs (50 ng) prepared using the RNeasy Micro Kit (Qiagen, Hilden, Germany) were reverse-transcribed to cDNA using Moloney Murine Leukemia Virus reverse transcriptase (Thermo Fisher Scientific) and random hexamers (Sigma-Aldrich). qRT-PCR was performed with a CFX96 Real-Time PCR

494 (Thermo Fisher Scientific). Gene expression was determined using the standard curve method.

495 Gene expression levels were normalized to those of Rn18s (18S ribosomal RNA). The primers 496 used for qRT-PCR are listed in Supplementary Table 5.

\section{In vitro testis reconstruction}

Fetal testes were reconstructed as previously described ${ }^{34,67}$. Briefly, $8.0 \times 10^{5}$ whole

500 testicular cells prepared from wild-type fetal testes at E16.5 were mixed with W-EGFP cells

$501\left(2.0 \times 10^{4}\right.$ cells $)$ at E16.5 or W-EGFP cells treated with siRNA $\left(2.5 \times 10^{4}\right.$ cells $)$. The

502 reconstructed testes were cultured on a V-shaped agarose gel for 2 days and then transferred

503 onto a bowl-shaped agarose gel, followed by culturing in $\alpha$-MEM containing $10 \%$ Knockout

504 Serum Replacement (Thermo Fisher Scientific) and PS at $37^{\circ} \mathrm{C}$ under $5 \% \mathrm{CO}_{2}$. To investigate 
505 the effect of HH and PDGF signals on FLC differentiation, the reconstructed tissues were

506 treated with SAG $(0.5 \mu \mathrm{M})$, mouse PDGF-AA $(10 \mathrm{ng} / \mathrm{mL})$, or DMSO. The reconstructed tissues

507 were observed under a BZ-X700 fluorescence microscope (Keyence) to capture EGFP-

508 fluorescent and bright-field images. The differentiation rate was calculated based on the EGFP-

509 positive area as previously described ${ }^{68}$.

\section{Western blotting analysis}

512 Whole-cell extracts were prepared from W-EGFP cells using lysis buffer (50 mM Tris-HCl

513 (pH 8.0), $50 \mathrm{mM} \mathrm{NaCl}, 1 \mathrm{mM}$ EDTA, and 1\% SDS) containing phosphatase inhibitors

514 (PhosSTOP tablet; Roche Diagnostics Corp., Indianapolis, IN, USA). After the protein

515 concentration was determined using a BCA Protein Assay Kit (Pierce Biotechnology, Rockford,

516 IL, USA), $5 \mu \mathrm{g}$ of the whole-cell extract was subjected to SDS-polyacrylamide gel

517 electrophoresis, followed by electrophoretic transfer to polyvinylidene fluoride membranes

518 (Thermo Fisher Scientific). The membranes were incubated for $30 \mathrm{~min}$ at room temperature in

519 Blocking One (Nacalai Tesque). The membranes were treated with primary antibodies in a

520 reaction buffer (10\% Blocking One in Tris-buffered saline; $10 \mathrm{mM}$ Tris- $\mathrm{HCl}(\mathrm{pH} 7.4), 150 \mathrm{mM}$

$521 \mathrm{NaCl}, 0.05 \%$ Tween 20 ) overnight at $4{ }^{\circ} \mathrm{C}$, and thereafter with horseradish peroxidase-

522 conjugated secondary antibodies in the reaction buffer for $1 \mathrm{~h}$ at room temperature. The primary

523 and secondary antibodies used in this study are listed in Supplementary Table 6. Washed

524 membranes were developed using Chemi-Lumi One (Nacalai Tesque), and the images were 
525 captured using a lumino image analyzer (ImageQuant LAS 500, GE Healthcare,

526 Buckinghamshire, UK). All images were quantified using Image Lab 6.0 software (Bio-Rad).

$528 \quad$ Preparation of expression plasmids and a donor plasmid

529 Full-length cDNAs of Tmsb10 and Kras was amplified by PCR with sets of primers

530 (Supplementary Table 7), and were used to construct p3xFLAG-TMSB10 and pCMV-HA-

531 KRAS expression plasmids, respectively. The p3xFLAG-CMV10 expression plasmid (Sigma-

532 Aldrich) was used as a control study.

533 To construct a donor plasmid for CRISPR/Cas9 technology, an 849-bp fragment upstream

534 from the first ATG and an 849-bp fragment downstream from the first ATG of the Tmsb10 gene

535 was amplified from the C57BL/6 genome. These fragments were used as the 5' and 3'

536 homologous arms. $m$ Cherry tagged with human influenza hemagglutinin (HA) and

537 Thoseaasigna virus 2A (T2A) at the $\mathrm{N}$ - and C-terminal sites (HA-mCherry-T2A), respectively,

538 was synthesized as follows. A DNA fragment encoding $m$ Cherry was amplified from

539 pmCherry-N1 (Clontech, Palo Alto, CA, USA) and inserted into the EcoRI/BglII site of pCMV-

540 HA (Takara, Shiga, Japan) to generate pCMV-HA-mCherry. Thereafter, the plasmid was

541 subjected to PCR with a 3' primer containing the T2A sequence to generate the HA-mCherry-

542 T2A fragment. The primers used for amplification of the fragments are listed in Supplementary

543 Table 7. Then, these three fragments (5' homologous arm, HA-mCherry-T2A, and 3' 
544 homologous arm) were inserted into the SalI/EcoRI site of the pBluescript II KS+ using an In-

545 Fusion HD cloning kit (Clontech) (Supplementary Fig. 3).

546

547 Physical interaction between TMSB10 and KRAS

p3xFLAG-TMSB10, p3xFLAG-CMV10, and pCMV-HA-KRAS were transfected using the

549 lipofectamine 2000 reagent (Thermo Fisher Scientific) in HEK 293 cells. The cells were lysed

550 in $50 \mathrm{mM}$ Tris- $\mathrm{HCl}(\mathrm{pH} 8.0), 300 \mathrm{mM} \mathrm{NaCl}, 10 \%$ glycerol, $1.5 \mathrm{mM} \mathrm{MgCl}_{2}, 1 \mathrm{mM}$ EDTA, and

$5511 \%$ Triton X-100. The p3xFLAG-TMSB10 and p3xFLAG-CMV10 were immunoprecipitated

552 with anti-FLAG antibody-conjugated magnetic beads (Sigma-Aldrich), and then the beads were

553 sequentially washed three times with washing buffer (20 mM HEPES-KOH (pH 7.6), $100 \mathrm{mM}$

$554 \mathrm{KCl}, 10 \%$ glycerol,1 mM EDTA, and $0.05 \%$ Tween-20), and once with PBS. Finally, the

555 immunoprecipitates were eluted from the beads with $10 \mu$ lelution buffer ( $50 \mathrm{mM}$ Tris- $\mathrm{HCl}(\mathrm{pH}$

556 7.4), $150 \mathrm{mM} \mathrm{NaCl}, 500 \mu \mathrm{g} / \mathrm{ml}$ FLAG peptide). Eluates and inputs were subjected to SDS-

557 polyacrylamide gel electrophoresis followed by Western blotting. The antibodies used in this

558 study are listed in Supplementary Table 6.

559

560 Generation of Tmsb10-mCherry knock-in mice

561

A mouse line carrying the $m$ Cherry reporter gene at the Tmsb10 locus (Tmsb10-mCherry

$562 \mathrm{KI}$ ) was generated using CRISPR/Cas9 technology ${ }^{69,70}$. A guide RNA (gRNA) was designed to

563 target the transcription start site of Tmsb10 using CRISPRdirect (http://crispr.dbcls.jp/). The 
564 gRNA was synthesized and purified using a CUGA7 gRNA Synthesis Kit (Nippon Gene,

565 Tokyo, Japan). Oocytes were collected from F1 hybrid (C57BL/6 x DBA/2) BDF1 female mice

566 that were superovulated by standard procedures and fertilized in vitro with sperms from male

567 mice of the same genetic background. CAS9 protein (100 ng/ $\mu \mathrm{l}$; Nippon Gene), the gRNA (250

$568 \mathrm{ng} / \mu \mathrm{l}$ each), and the donor plasmid were microinjected into the cytoplasm on one side of the

569 blastomere at the two-cell stage. The cells were transferred to pseudo-pregnant ICR female

570 mice. Genotypes of the pups were analyzed by PCR. Nucleotide sequences of the primers for

571 gRNA preparation and genotyping are shown in Supplementary Table 7. After backcrossing

572 more than five times with C57BL/6J, Tmsb10-m Cherry knock-in mice were further crossed

573 with FLE-EGFP mice to generate FLE-EGFP;Tmsb10-mCherry mice. FLE-EGFP and FLE-

574 EGFP;Tmsb10-m Cherry male mice were crossed with ICR females (Japan SLC, Shizuoka, 575 Japan).

576

577 Statistical analyses

578 At least three biologically independent samples were used in all experiments. All data are

579 presented as mean \pm standard error of the mean (SEM). We used Student's $t$-tests for

580 comparisons between two groups, and one-way analysis of variance (ANOVA) followed by

581 Tukey's multiple comparison test for multiple group comparisons. $p<0.05$ was considered to

582 indicate statistically significant differences between groups. The statistical analyses were

583 performed using R software version 3.6.3 (https://www.r-project.org). 


\section{REFERENCES}

5851 Svingen, T. \& Koopman, P. Building the mammalian testis: origins, differentiation, and 586 assembly of the component cell populations. Genes \& development 27, 2409-2426, 587 doi:10.1101/gad.228080.113(2013).

5882 Zirkin, B. R. \& Papadopoulos, V. Leydig cells: formation, function, and regulation. 589 Biology of reproduction 99, 101-111, doi:10.1093/biolre/ioy059 (2018).

5903 Yao, H. H., Whoriskey, W. \& Capel, B. Desert Hedgehog/Patched 1 signaling specifies 591 fetal Leydig cell fate in testis organogenesis. Genes \& development 16, 1433-1440, 592 doi:10.1101/gad.981202 (2002).

5934 Brennan, J., Tilmann, C. \& Capel, B. Pdgfr-alpha mediates testis cord organization and 594 fetal Leydig cell development in the XY gonad. Genes \& development 17, 800-810, doi:10.1101/gad.1052503 (2003).

5965 Tang, H. et al. Notch signaling maintains Leydig progenitor cells in the mouse testis. Development 135, 3745-3753, doi:10.1242/dev.024786 (2008).

5986 Sarraj, M. A. et al. Fetal Testis Dysgenesis and Compromised Leydig Cell Function in 599 Tgfbr3 (Betaglycan) Knockout Mice1. Biology of reproduction 82, 153-162, 600 doi:10.1095/biolreprod.109.078766 (2010).

6017 Kitamura, K. et al. Mutation of ARX causes abnormal development of forebrain and 602 testes in mice and X-linked lissencephaly with abnormal genitalia in humans. Nature 603 genetics 32, 359-369, doi:10.1038/ng1009 (2002). 
6048 Cui, S. et al. Disrupted gonadogenesis and male-to-female sex reversal in Pod1

605 knockout mice. Development 131, 4095-4105, doi:10.1242/dev.01266 (2004).

6069 Buaas, F. W., Gardiner, J. R., Clayton, S., Val, P. \& Swain, A. In vivo evidence for the 607 crucial role of SF1 in steroid-producing cells of the testis, ovary and adrenal gland.

608 Development 139, 4561-4570, doi:10.1242/dev.087247 (2012).

60910 Shima, Y. et al. Fetal Leydig cells dedifferentiate and serve as adult Leydig stem cells.

$610 \quad$ Development 145, doi:10.1242/dev.169136 (2018).

61111 Barsoum, I. \& Yao, H. H. Redundant and differential roles of transcription factors Gli1

612 and Gli2 in the development of mouse fetal Leydig cells. Biology of reproduction 84,

613 894-899, doi:10.1095/biolreprod.110.088997 (2011).

61412 Kothandapani, A. et al. GLI3 resides at the intersection of hedgehog and androgen

615 action to promote male sex differentiation. PLoS genetics 16, e1008810,

616 doi:10.1371/journal.pgen.1008810(2020).

61713 Miyabayashi, K. et al. Aristaless related homeobox gene, Arx, is implicated in mouse

618 fetal Leydig cell differentiation possibly through expressing in the progenitor cells.

$619 \quad$ PLoS one 8, e68050, doi:10.1371/journal.pone.0068050 (2013).

62014 DeFalco, T., Takahashi, S. \& Capel, B. Two distinct origins for Leydig cell progenitors 621 in the fetal testis. Developmental biology 352, 14-26, doi:10.1016/j.ydbio.2011.01.011

622 (2011). 
62315 Defalco, T., Saraswathula, A., Briot, A., Iruela-Arispe, M. L. \& Capel, B. Testosterone 624 levels influence mouse fetal Leydig cell progenitors through notch signaling. Biology of 625 reproduction $\mathbf{8 8}, 91$, doi:10.1095/biolreprod.112.106138 (2013).

62616 Liu, C., Rodriguez, K. \& Yao, H. H. Mapping lineage progression of somatic progenitor 627 cells in the mouse fetal testis. Development 143, 3700-3710, doi:10.1242/dev.135756 $628 \quad$ (2016).

62917 Kumar, D. L. \& DeFalco, T. A perivascular niche for multipotent progenitors in the 630 fetal testis. Nature communications 9, 4519, doi:10.1038/s41467-018-06996-3 (2018).

63118 Stevant, I. et al. Deciphering Cell Lineage Specification during Male Sex

632 Determination with Single-Cell RNA Sequencing. Cell reports 22, 1589-1599, 633 doi:10.1016/j.celrep.2018.01.043 (2018).

63419 Ademi, H., Stévant, I., Rands, C. M., Conne, B. \& Nef, S. Expression of Wnt5a defines 635 the major progenitors of fetal and adult Leydig cells. bioRxiv, $636 \quad$ doi:10.1101/2020.07.25.221069 (2020).

63720 Bangs, F. \& Anderson, K. V. Primary Cilia and Mammalian Hedgehog Signaling. Cold 638 Spring Harbor perspectives in biology 9, doi:10.1101/cshperspect.a028175 (2017).

63921 Hui, C. C. \& Angers, S. Gli proteins in development and disease. Annual review of cell 640 and developmental biology 27, 513-537, doi:10.1146/annurev-cellbio-092910-154048 641 (2011). 
64222 Andrae, J., Gallini, R. \& Betsholtz, C. Role of platelet-derived growth factors in

643 physiology and medicine. Genes \& development 22, 1276-1312,

644 doi:10.1101/gad.1653708 (2008).

64523 Goldstein, A. L., Slater, F. D. \& White, A. Preparation, assay, and partial purification of 646 a thymic lymphocytopoietic factor (thymosin). Proceedings of the National Academy of 647 Sciences of the United States of America 56, 1010-1017, doi:10.1073/pnas.56.3.1010 $648 \quad$ (1966).

64924 Lin, S. C. \& Morrison-Bogorad, M. Developmental expression of mRNAs encoding 650 thymosins beta 4 and beta 10 in rat brain and other tissues. Journal of molecular 651 neuroscience : MN 2, 35-44, doi: 10.1007/BF02896924 (1990).

65225 Carpintero, P., Franco del Amo, F., Anadón, R. \& Gómez-Márquez, J. Thymosin 653 beta10 mRNA expression during early postimplantation mouse development. FEBS 654 letters 394, 103-106, doi:10.1016/0014-5793(96)00888-5 (1996).

65526 Hannappel, E. beta-Thymosins. Annals of the New York Academy of Sciences 1112, 21$656 \quad 37$, doi:10.1196/annals.1415.018 (2007).

65727 Safer, D., Elzinga, M. \& Nachmias, V. T. Thymosin beta 4 and Fx, an actin658 sequestering peptide, are indistinguishable. The Journal of biological chemistry 266, 659 4029-4032 (1991). 
66028 Yu, F. X., Lin, S. C., Morrison-Bogorad, M., Atkinson, M. A. \& Yin, H. L. Thymosin 661 beta 10 and thymosin beta 4 are both actin monomer sequestering proteins. The Journal $662 \quad$ of biological chemistry 268, 502-509 (1993).

66329 Sribenja, S., Wongkham, S., Wongkham, C., Yao, Q. \& Chen, C. Roles and 664 mechanisms of beta-thymosins in cell migration and cancer metastasis: an update.

$665 \quad$ Cancer investigation 31, 103-110, doi:10.3109/07357907.2012.756111 (2013).

$66630 \quad$ Lee, S. H. et al. Thymosin $\beta 10$ inhibits angiogenesis and tumor growth by interfering 667 with Ras function. Cancer research 65, 137-148 (2005).

66831 Shima, Y. et al. Identification of an enhancer in the Ad4BP/SF-1 gene specific for fetal 669 Leydig cells. Endocrinology 153, 417-425, doi:10.1210/en.2011-1407 (2012).

67032 Shima, Y. et al. Contribution of Leydig and Sertoli cells to testosterone production in 671 mouse fetal testes. Molecular endocrinology 27, 63-73, doi:10.1210/me.2012-1256 $672 \quad(2013)$.

67333 Orth, J. M. Proliferation of Sertoli cells in fetal and postnatal rats: a quantitative 674 autoradiographic study. The Anatomical record 203, 485-492, 675 doi:10.1002/ar.1092030408 (1982).

67634 Inoue, M. et al. Isolation and Characterization of Fetal Leydig Progenitor Cells of Male 677 Mice. Endocrinology 157, 1222-1233, doi:10.1210/en.2015-1773 (2016).

67835 Hashimshony, T. et al. CEL-Seq2: sensitive highly-multiplexed single-cell RNA-Seq. 679 Genome biology 17, 77, doi:10.1186/s13059-016-0938-8 (2016). 
68036 Nef, S. \& Parada, L. F. Cryptorchidism in mice mutant for Insl3. Nature genetics 22,

$681 \quad$ 295-299, doi:10.1038/10364 (1999).

68237 Zimmermann, S. et al. Targeted Disruption of the Ins13 Gene Causes Bilateral

683 Cryptorchidism. Molecular endocrinology 13, 681-691, doi:10.1210/mend.13.5.0272

$684 \quad(1999)$

68538 Anderson, C. M. \& Mendelson, C. R. Developmental and hormonal regulation of

686 cholesterol side chain cleavage cytochrome P-450 in the fetal rabbit testis. Molecular

687 and cellular endocrinology 55, 121-130, doi:10.1016/0303-7207(88)90126-8 (1988).

68839 O'Shaughnessy, P. J., Willerton, L. \& Baker, P. J. Changes in Leydig cell gene

689 expression during development in the mouse. Biology of reproduction 66, 966-975, doi:

$690 \quad 10.1095 /$ biolreprod66.4.966 (2002).

$69140 \quad$ Morohashi, K., Baba, T. \& Tanaka, M. Steroid hormones and the development of

692 reproductive organs. Sexual development 7, 61-79, doi:10.1159/000342272 (2013).

69341 Morohashi, K. I. \& Omura, T. Ad4BP/SF-1, a transcription factor essential for the

694 transcription of steroidogenic cytochrome P450 genes and for the establishment of the

695 reproductive function. FASEB journal 10, 1569-1577, doi:

$696 \quad$ 10.1096/fasebj.10.14.9002548 (1996).

69742 Parker, K. L. \& Schimmer, B. P. Steroidogenic factor 1: a key determinant of endocrine 698 development and function. Endocrine reviews 18, 361-377, doi:10.1210/edrv.18.3.0301

699 (1997). 
70043 Kasahara, K. \& Inagaki, M. Primary ciliary signaling: links with the cell cycle. Trends in cell biology 31, 954-964, doi:10.1016/j.tcb.2021.07.009 (2021).

70244 Wang, S., Wei, Q., Dong, G. \& Dong, Z. ERK-mediated suppression of cilia in cisplatin-induced tubular cell apoptosis and acute kidney injury. Biochimica et biophysica acta 1832, 1582-1590, doi:10.1016/j.bbadis.2013.05.023 (2013).

$70545 \quad$ Jenks, A. D. et al. Primary Cilia Mediate Diverse Kinase Inhibitor Resistance

706 Mechanisms in Cancer. Cell reports 23, 3042-3055, doi:10.1016/j.celrep.2018.05.016 $707 \quad$ (2018).

$70846 \quad$ Kobayashi, T. et al. HDAC2 promotes loss of primary cilia in pancreatic ductal adenocarcinoma. EMBO reports 18, 334-343, doi:10.15252/embr.201541922 (2017).

$71047 \quad$ Ying, H. Z. et al. PDGF signaling pathway in hepatic fibrosis pathogenesis and 711 therapeutics (Review). Molecular medicine reports 16, 7879-7889, 712 doi:10.3892/mmr.2017.7641 (2017).

71348 Chalhoub, N. \& Baker, S. J. PTEN and the PI3-kinase pathway in cancer. Annual 714 review of pathology 4, 127-150, doi:10.1146/annurev.pathol.4.110807.092311 (2009).

71549 Rommel, C. et al. Differentiation stage-specific inhibition of the Raf-MEK-ERK

716 pathway by Akt. Science 286, 1738-1741, doi:10.1126/science.286.5445.1738 (1999).

$71750 \quad$ Hwang, B., Lee, J. H. \& Bang, D. Single-cell RNA sequencing technologies and 718 bioinformatics pipelines. Experimental \& molecular medicine 50, 1-14, 719 doi:10.1038/s12276-018-0071-8 (2018). 
72051 Green, C. D. et al. A Comprehensive Roadmap of Murine Spermatogenesis Defined by Single-Cell RNA-Seq. Developmental cell 46, 651-667.e610, doi:10.1016/j.devcel.2018.07.025 (2018).

72352 Hermann, B. P. et al. The Mammalian Spermatogenesis Single-Cell Transcriptome,

724 from Spermatogonial Stem Cells to Spermatids. Cell reports 25, 1650-1667.e1658, doi:10.1016/j.celrep.2018.10.026 (2018).

$72653 \quad$ Lukassen, S., Bosch, E., Ekici, A. B. \& Winterpacht, A. Single-cell RNA sequencing of adult mouse testes. Scientific data 5, 180192, doi:10.1038/sdata.2018.192 (2018).

72854 Jung, M. et al. Unified single-cell analysis of testis gene regulation and pathology in 729 five mouse strains. eLife 8, doi:10.7554/eLife.43966 (2019).

73055 Tan, K., Song, H. W. \& Wilkinson, M. F. Single-cell RNAseq analysis of testicular 731 germ and somatic cell development during the perinatal period. Development 147, doi:10.1242/dev.183251 (2020).

73356 Shiotsuka, M. et al. The expression and function of thymosin beta 10 in tooth germ 734 development. The International journal of developmental biology 57, 873-883, 735 doi:10.1387/ijdb.120240hs (2013).

73657 Zhang, W. et al. Deer thymosin beta 10 functions as a novel factor for angiogenesis and 737 chondrogenesis during antler growth and regeneration. Stem cell research \& therapy $\mathbf{9}$, 738 166-166, doi:10.1186/s13287-018-0917-y (2018). 
73958 Salhab, M. et al. Thymosins $\beta-4$ and $\beta-10$ are expressed in bovine ovarian follicles and 740 upregulated in cumulus cells during meiotic maturation. Reproduction, Fertility and Development 22, 1206-1221, doi:10.1071/RD10015 (2010).

74259 Anadón, R. et al. Differential expression of thymosins beta(4) and beta(10) during rat cerebellum postnatal development. Brain Research 894, 255-265, doi:10.1016/s00068993(01)02024-8 (2001).

745

60 Zhang, X. et al. Thymosin beta 10 is a key regulator of tumorigenesis and metastasis and a novel serum marker in breast cancer. Breast Cancer Research 19, 15-15, doi:10.1186/s13058-016-0785-2 (2017). osteogenic differentiation of human mesenchymal stem cells in a TGF- $\beta$-activated MEK-dependent manner. International journal of molecular medicine 33, 534-542, doi:10.3892/ijmm.2013.1606 (2014). signaling. PLoS biology 17, e3000133, doi:10.1371/journal.pbio.3000133 (2019).

75463 Langmead, B. \& Salzberg, S. L. Fast gapped-read alignment with Bowtie 2. Nature methods 9, 357-359, doi:10.1038/nmeth.1923 (2012).

75664 Anders, S., Pyl, P. T. \& Huber, W. HTSeq--a Python framework to work with highthroughput sequencing data. Bioinformatics 31, 166-169, doi:10.1093/bioinformatics/btu638 (2015). 
75965 Lun, A. T., McCarthy, D. J. \& Marioni, J. C. A step-by-step workflow for low-level

760 analysis of single-cell RNA-seq data with Bioconductor. F1000Research 5, 2122,

761 doi:10.12688/f1000research.9501.2 (2016).

76266 Yanai, S. et al. Gene expression and functional abnormalities in XX/Sry Leydig cells.

763 Scientific reports 11, 719, doi:10.1038/s41598-020-80741-z (2021).

76467 Yokonishi, T. et al. In Vitro Reconstruction of Mouse Seminiferous Tubules Supporting

765 Germ Cell Differentiation. Biology of reproduction 89 1-6,

766 doi:10.1095/biolreprod.113.108613 (2013).

76768 Fukunaga, H. et al. High-precision microbeam radiotherapy reveals testicular tissue-

768 sparing effects for male fertility preservation. Scientific reports $\mathbf{9}, 12618$

769 doi:10.1038/s41598-019-48772-3 (2019).

77069 Jinek, M. et al. A programmable dual-RNA-guided DNA endonuclease in adaptive

771 bacterial immunity. Science 337, 816-821, doi:10.1126/science.1225829 (2012).

77270 Hara, S., Terao, M., Muramatsu, A. \& Takada, S. Efficient production and transmission

773 of CRISPR/Cas9-mediated mutant alleles at the IG-DMR via generation of mosaic mice

774 using a modified 2CC method. Scientific reports 9, 20202, doi:10.1038/s41598-019-

775

56676-5 (2019). 
777 M.I., T.B., Y.S., and K.-I.M. conceived, designed, and conducted the experiments, and performed

778 data analyses. F.T. and Y.O. constructed the scRNA-seq libraries and obtained transcriptomes.

779 M.I., S.Y., D.S., and M.S. analyzed the sequence data. M.T. and S.T. produced the genome-edited

780 mice. K.S. and T.M. analyzed cell ciliation. M.I. and K.-I.M. prepared the manuscript.

781

782 Acknowledgements

783 We thank Dr. Junko Sasaki (Medical Research Institute, Tokyo Medical and Dental University)

784 for her technical advice and discussion. We appreciate the technical assistance of The Research

785 Support Center, Research Center for Human Disease Modeling, Kyushu University Graduate

786 School of Medical Sciences. This work was supported by JSPS KAKENHI Grant Numbers

787 JP20K08863 (T.B.), JP17H06427 (T.B., K.-I.M.), JP20H03436 (K.-I.M.), JP20H04935 (S.T.),

788 JP19H05244 (Y.O.), JP20H00456 (Y.O.), JP20H04846 (Y.O.), and JP20K21398 (Y.O.); by JST

789 CREST Grant Number JPMJCR16G1 (Y.O.); and by AMED under Grant Numbers

790 JP20gk0210019 (K.-I.M.) and JP20ek0109489h0001 (Y.O.).

791

792 Author information

793 scRNA-seq data have been deposited in the DNA Data Bank of Japan under the accession code

794 DRA013467 (http://trace.ddbj.nig.ac.jp/DRASearch/). 
796 Figure 1. Characterization of S- and W-EGFP cells by scRNA-seq

797 (a) High-quality scRNA-seq datasets obtained from 80 S-EGFP and 341 W-EGFP cells were

798 analyzed by hierarchical clustering. As indicated by the dendrogram (upper), the cells were

799 divided into five cell clusters: cluster A (204 W-EGFP cells; light blue), cluster B (80 W-EGFP

800 cells; blue), cluster C (57 W-EGFP cells; orange), cluster D (13 S-EGFP cells; green), and cluster

801 E (67 S-EGFP cells; red). The heat map (lower) was based on genes in clusters C, D, and/or E

802 whose expressions were altered. Genes differentially expressed in these clusters (group I to III)

803 are shown. Tmsb10 in group III is highlighted in red. $(\boldsymbol{b}, \boldsymbol{c})$ Results of t-SNE (b) and Monocle

804 pseudo-time trajectory analyses (c) of the datasets are shown. The cellular distribution is shown

805 (each dot represents one cell), with colors labeling the five clusters of cells as in (a). (d-g)

806 Expression levels of Cyplla1 (d), Calr (e), Tmsb10 (f), and Arx (g) in the cells above, as

807 determined by scRNA-seq. Each dot represents one cell.

808

809 Figure 2. Increased expression of Tmsb10 in putative FLC progenitors

810 (a) The testes of FLE-EGFP;Tmsb10-mCherry mouse fetuses at E16.5 were analyzed by

811 immunofluorescence. Representative images of staining with EGFP (green), mCherry (red), and

812 LAMININ (white), with all three stains overlaid in the image on the right. Arrows indicate cells

813 double positive for mCherry and LAMININ. Closed arrowheads indicate cells double positive for

814 mCherry (weakly stained) and EGFP. Open arrowheads indicate FLCs strongly stained with 
815 EGFP. Asterisks mark testis tubules. Scale bar $=20 \mu \mathrm{m}$. (b-d) Expressions of EGFP (b), Tmsb10

816 (c), and Pecaml (d) in N-EGFP (EGFP-negative, N, open bars), W-EGFP (W, light blue bars),

817 and S-EGFP (S, blue bars) cells prepared from FLE-EGFP fetal testes at E16.5. The data were

818 normalized by $R n 18 s$ and are presented as means \pm SEM. Letters a, b, and c denote significant

819 differences between the cell groups, N-EGFP (N), W-EGFP (W), and S-EGFP (S). $n=3 \cdot p<0.01$.

\section{$821 \quad$ Figure 3. Suppression of FLC differentiation by Tmsb10 KD}

822 (a) The experimental procedure for reconstruction of fetal testes. W-EGFP cells (E16.5) were

823 plated and treated with siRNA for $24 \mathrm{~h}$. Whole testicular cells prepared from wild-type fetuses

824 (E16.5) were mixed with siRNA-treated W-EGFP cells to reconstruct testes. The detailed

825 procedure for testis reconstruction and culture is described in the Materials and Methods. (b) A

826 representative image of the reconstructed testis. Testicular tubules (tu) formed, and FLCs with

827 strong EGFP staining were observed in the interstitial regions of the reconstructed testes. (c) The

828 reconstructed testes were cultured in the presence $(+)$ or absence (-) of SAG for 21 days. The

829 reconstructed testes were examined under a fluorescence microscope to measure EGFP

830 fluorescence. Scale bar $=100 \mu \mathrm{m}$. (d) The EGFP-positive cells (indicated by the relative EGFP-

831 positive area) in the reconstructed testes above were analyzed quantitatively after incubation for

83221 days. The cells were cultured in the absence (-, open bar) or presence of SAG (+, gray bar).

$833 n=3$. ${ }^{* * *} p<0.001$. (e) Representative images of the reconstructed testes using W-EGFP cells

834 treated with the following siRNAs: control (siCnt), Tmsb10 (siT10), Tmsb4x (siT4x), and 
$835 A d 4 B P / S F-1($ si $A d 4)$. Scale bar $=100 \mu \mathrm{m} .(f)$ The EGFP-positive cells in the reconstructed testes

836 above were analyzed quantitatively. Letters $a, b$, and $\mathrm{c}$ denote significant differences between the

837 cell groups treated with siCnt, siT10, siT4x, and siAd4. $n=3 . p<0.001$. (g) Testes were

838 reconstructed using W-EGFP cells treated with siCnt or siT10. They were cultured for 21 days in

839 the presence of SAG. Scale bar $=100 \mu \mathrm{m}$. (h) The EGFP-positive cells in the reconstructed testes

840 above were analyzed quantitatively. $n=3 .{ }^{* * *} p<0.001$. (i) W-EGFP cells were treated with siCnt

841 (open bar) or siT10 (light blue bar). Expression of Gli1 in the W-EGFP cells was examined by

842 qRT-PCR. The data were normalized by Rn18s and are presented as means \pm SEM. $n=3$.

$843 * * p<0.01$.

844

845 Figure 4. Role of Tmsb10 in the regulation of ciliogenesis via suppression of the RAS/ERK

846 pathway

847 (a) E16.5 W-EGFP cells were treated with $0.1,1.0$, or $10.0 \mathrm{nM}$ siT10 or $10.0 \mathrm{nM}$ siCnt, then

848 cultured in serum-free medium for $24 \mathrm{~h}$. Thereafter, the cells were subjected to immunostaining

849 for the cilial marker protein ARL13B (red). Nuclei were stained with 4',6'-diamidino-2-

850 phenylindole (DAPI) (blue). The enclosed area is enlarged at the top right. Arrows indicate

851 primary cilia. Scale bar $=10 \mu \mathrm{m}$. (b) Ciliated cells detected in the studies above were counted.

852 The ratios of the ciliated cells to all cells (\%) are shown. $n=5 .{ }^{*} p<0.001$. (c) W-EGFP cells were

853 treated with $0.1,1.0$, or $10.0 \mathrm{nM}$ siT10 or $10.0 \mathrm{nM}$ siCnt, then subjected to testis reconstruction

854 assay. The EGFP-positive cells (indicated by the relative EGFP-positive area) in the reconstructed 
855 testes were analyzed quantitatively after incubation for 21 days. $n=3 \cdot p<0.001$. (d) W-EGFP 856 cells were cultured and treated with $0.1,1.0$, or $10.0 \mathrm{nM}$ siT10 or $10.0 \mathrm{nM}$ siCnt. Expression of 857 the Gli1 gene in the cells was examined. The data were standardized using Rn18s. $n=3 . p<0.001$.

858 (e) Interactions between TMSB10 and RAS were examined. Whole-cell lysates were prepared 859 from HEK293 cells overexpressing FLAG-TMSB10 or FLAG together with KRAS. Proteins 860 interacting with TMSB10 were immunoprecipitated with anti-FLAG antibody. The 861 immunoprecipitates were subjected to immunoblotting using antibodies for pan-RAS and FLAG.

862 The positions of molecular weight markers are indicated at the left. Full blot images are displayed 863 in Supplemental Fig. 8. (f) Whole-cell extracts were prepared from E16.5 W-EGFP cells treated 864 with siT10 or siCnt in the presence $(+)$ or absence (-) of SAG. Levels of phospho-ERK (pERK) 865 and ERK were examined by Western blotting. (g) Signal intensities in the blots above were 866 quantified as described in the Materials and Methods. The amounts of pERK relative to ERK are 867 shown. $n=3$. $p<0.01$. (h) A schematic illustration summarizing the results so far. TMSB10 was 868 assumed to suppress the RAS/ERK pathway by interacting with RAS. (i) W-EGFP cells were 869 treated with siCnt (open bar), siT10 (light blue bar), siRas (dark blue bar), or both siT10 and siRas 870 (red bar). These cells were then used for testis reconstruction. The reconstructed testes were 871 cultured in the presence of SAG for 21 days. The EGFP-positive cells in the reconstructed testes 872 were analyzed quantitatively. $n=3 \cdot p<0.001$. (j) W-EGFP cells were treated with siRNAs as 873 above and then cultured in the presence of SAG. The expression of Glil was examined by qRT874 PCR. The data were standardized using Rn18s. $n=3$. $p<0.001$. ( $\boldsymbol{k}$ ) W-EGFP cells were treated 
875 with siRNAs as above, and their effect on ciliogenesis was examined by immunostaining for

876 ARL13B. Ciliated cells were counted and the ratios of these cells to all cells (\%) are plotted. $n=$ 877 5. ${ }^{*} p<0.001$. Letters $\mathrm{a}, \mathrm{b}$, and $\mathrm{c}$ on the bars in $(c),(d),(g),(i)$, and $(j)$ denote significant differences 878 between the cell groups.

\section{Figure 5. Activation of the RAS/ERK pathway by PDGF}

881 (a) Reconstructed testes were incubated in the absence (open bar) or presence of PDGF-AA

882 (green bar), SAG (dark blue bar), or both PDGF-AA and SAG (red bar). The EGFP-positive cells

883 in the reconstructed testes were analyzed quantitatively after incubation for 21 days. $n=3$.

$884 p<0.001$. (b) W-EGFP cells were cultured under the same conditions as above. Expression of Gli1

885 in the cells was examined by qRT-PCR. The data were normalized by Rn18s and are presented

886 as means \pm SEM. $n=3$. $p<0.01$. (c) Whole-cell extracts prepared from W-EGFP cells were

887 cultured in the presence (+) or absence (-) of PDGF-AA and subjected to Western blotting for

888 ERK and pERK. The position of a molecular weight marker is indicated at the left. (d) Signal

889 intensities of the blots above were quantified as described in the Materials and Methods. The

890 amounts of pERK relative to ERK are shown. Letters a, b, and c on the bars in (a), (b), and (d)

891 denote significant differences between the cell groups. $n=3 . p<0.01$. (e) W-EGFP cells were

892 treated with siRNA for siT10 and thereafter cultured in the presence $(+)$ or absence $(-)$ of PDGF-

893 AA. Whole-cell extracts prepared from the cells were subjected to Western blotting for ERK and

894 pERK. (f) Signal intensities of the blots above were quantified. The amounts of pERK relative to 
895 ERK are shown. $n=3 .{ }^{* * *} p<0.001$. Full blot images for (c) and (d) are displayed in Supplemental

896 Fig. 8. (g) A tentative schema for the function of PDGF is shown. It was assumed that another

897 signal pathway could be activated downstream of PDGF. (h) W-EGFP cells were cultured in the

898 absence (-) or presence $(+)$ of PDGF-AA and SAG. Ciliogenesis in the W-EGFP cells was

899 examined by immunostaining for ARL13B. Ciliated cells were counted and the ratios of these

900 cells to all cells (\%) are plotted. $n=3 . * p<0.001$.

901

902 Figure 6. Dual role of PDGF in regulating FLC differentiation

903 (a) A schematic summary of signal pathways regulating FLC differentiation and in which Tmsb10

904 acts as a suppressor. (b) W-EGFP cells were cultured in the presence (+) or absence (-) of PDGF-

905 AA. Whole-cell extracts prepared from the cells were subjected to Western blotting for phospho-

906 AKT (pAKT) and AKT. Representative images of the blots are shown. The positions of molecular

907 weight markers are indicated at the left. (c) Signal intensities in the blots above were quantified

908 as described in the Materials and Methods. The amounts of pAKT relative to AKT are shown. $n$

$909=3 .{ }^{*} p<0.05$. (d) W-EGFP cells were treated with siCnt, siT10, siAkt, or siPten, and then used

910 for testis reconstruction. The reconstructed testes were cultured in the presence of SAG and PDGF

911 for 21 days. The EGFP-positive cells in the reconstructed testes were quantified. $n=3 . p<0.001$.

912 (e) W-EGFP cells were cultured under the same conditions as above. Expression of Glil in the

913 cells was determined by qRT-PCR. The data were normalized by $R n 18 s$ and are presented as

914 means \pm SEM. $n=3 . p<0.001$. Letters $\mathrm{a}, \mathrm{b}$, and $\mathrm{c}$ on the bars in $(d)$ and (e) denote significant 
915 differences. $(f)$ W-EGFP cells were cultured under the same conditions as above. Ciliogenesis in

916 the W-EGFP cells was examined by immunostaining for ARL13B. $n=5 .{ }^{*} p<0.001$. (g) W-EGFP

917 cells were cultured in the presence (+) or absence (-) of wortmannin (Wort). Whole-cell extracts

918 were subjected to Western blotting for pAKT, AKT, pERK, and ERK. (h) Signal intensities in

919 the blots above were quantified as described in the Materials and Methods. The amounts of pAKT

920 relative to AKT (left) and pERK relative to ERK (right) are shown. $n=3 .{ }^{* *} p<0.01 .{ }^{* * *} p<0.001$.

921 (i) W-EGFP cells treated with siRNA for siPten and siCnt. Whole-cell extracts prepared from the

922 cells were subjected to Western blotting for pAKT, AKT, pERK, and ERK. Full blot images for

923 (b), (g), and (i) are shown in Supplemental Fig. 8. (j) Signal intensities in the blots above were

924 quantified. The amounts of pAKT relative to AKT (left) and pERK relative to ERK (right) are

925 shown. $n=3 .{ }^{*} p<0.05 .{ }^{* *} p<0.01$. 

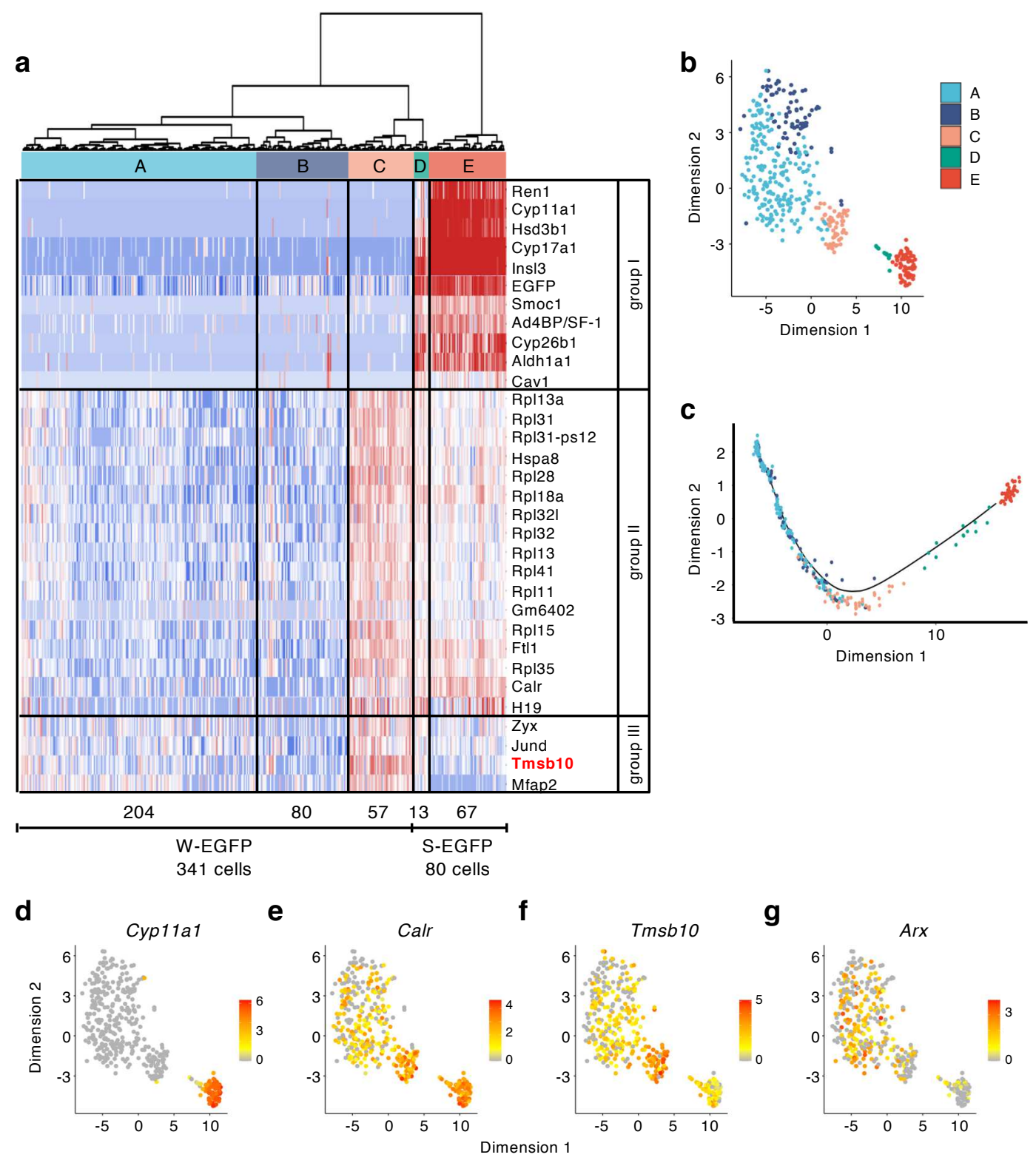

Figure 1. Characterization of S- and W-EGFP cells by scRNA-seq

(a) High-quality scRNA-seq datasets obtained from 80 S-EGFP and 341 W-EGFP cells were analyzed by hierarchical clustering. As indicated by the dendrogram (upper), the cells were divided into five cell clusters: cluster A (204 W-EGFP cells; light blue), cluster B (80 W-EGFP cells; blue), cluster C (57 W-EGFP cells; orange), cluster D (13 S-EGFP cells; green), and cluster E (67 S-EGFP cells; red). The heat map (lower) was based on genes in clusters $C, D$, and/or $E$ whose expressions were altered. Genes differentially expressed in these clusters (group I to III) are shown. Tmsb10 in group III is highlighted in red. $(\boldsymbol{b}, \boldsymbol{c})$ Results of t-SNE (b) and Monocle pseudo-time trajectory analyses (c) of the datasets are shown. The cellular distribution is shown (each dot represents one cell), with colors labeling the five clusters of cells as in (a). (d-g) Expression levels of Cyp11a1 (d), Calr (e), Tmsb10 (f), and Arx (g) in the cells above, as determined by scRNA-seq. Each dot represents one cell. 
a
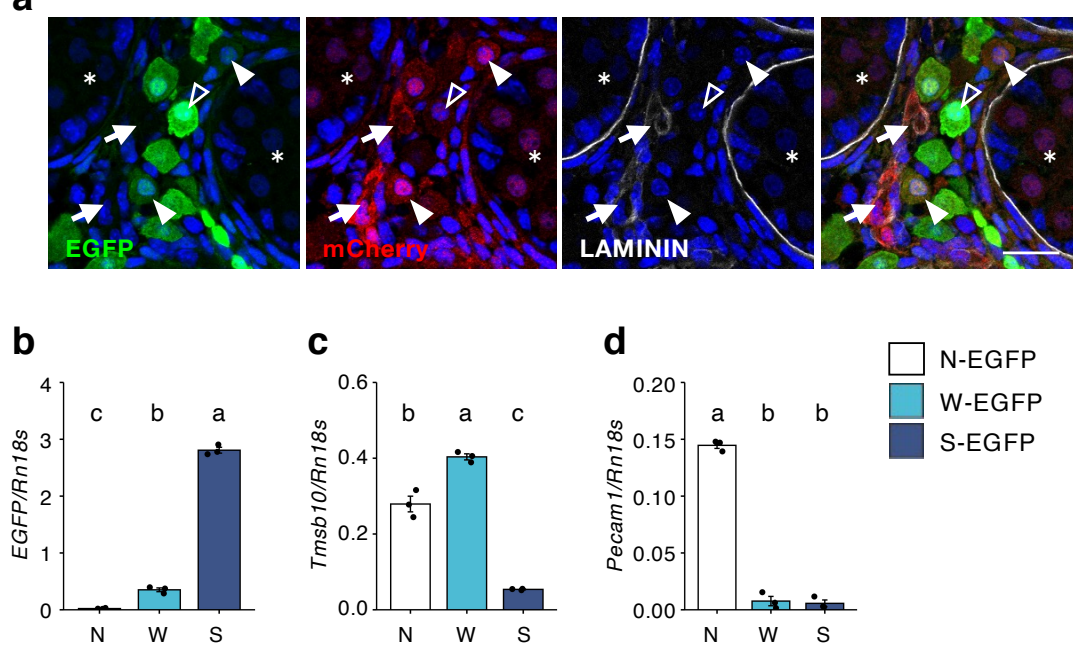

Figure 2. Increased expression of Tmsb10 in putative FLC progenitors

(a) The testes of FLE-EGFP;Tmsb10-mCherry mouse fetuses at E16.5 were analyzed by immunofluorescence. Representative images of staining with EGFP (green), mCherry (red), and LAMININ (white), with all three stains overlaid in the image on the right. Arrows indicate cells double positive for mCherry and LAMININ. Closed arrowheads indicate cells double positive for mCherry (weakly stained) and EGFP. Open arrowheads indicate FLCs strongly stained with EGFP. Asterisks mark testis tubules. Scale bar $=20 \mu \mathrm{m}$. (b-d) Expressions of EGFP (b), Tmsb10 (c), and Pecam1 (d) in N-EGFP (EGFP-negative, N, open bars), W-EGFP (W, light blue bars), and S-EGFP (S, blue bars) cells prepared from FLE-EGFP fetal testes at E16.5. The data were normalized by Rn18s and are presented as means \pm SEM. Letters $a, b$, and $c$ denote significant differences between the cell groups, N-EGFP (N), W-EGFP (W), and S-EGFP (S). $n=3 . p<0.01$. 
a

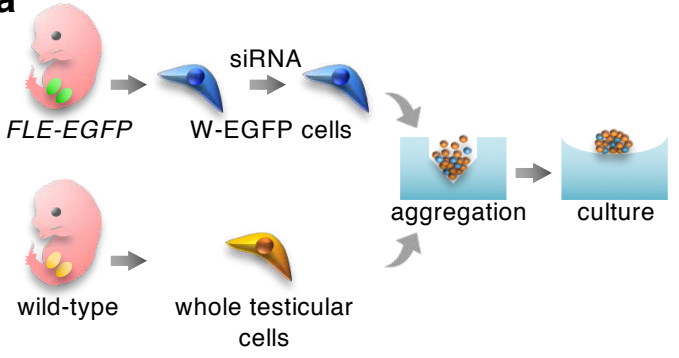

b

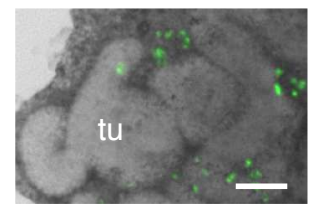

c

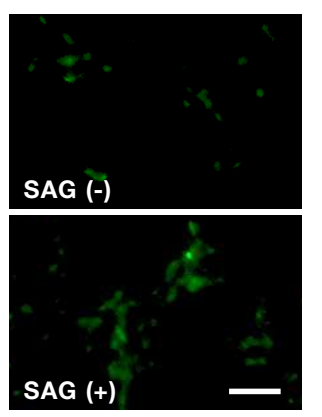

d

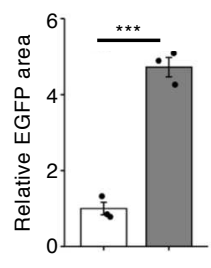

SAG - +

e
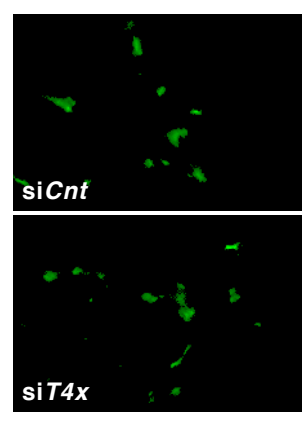

siT10
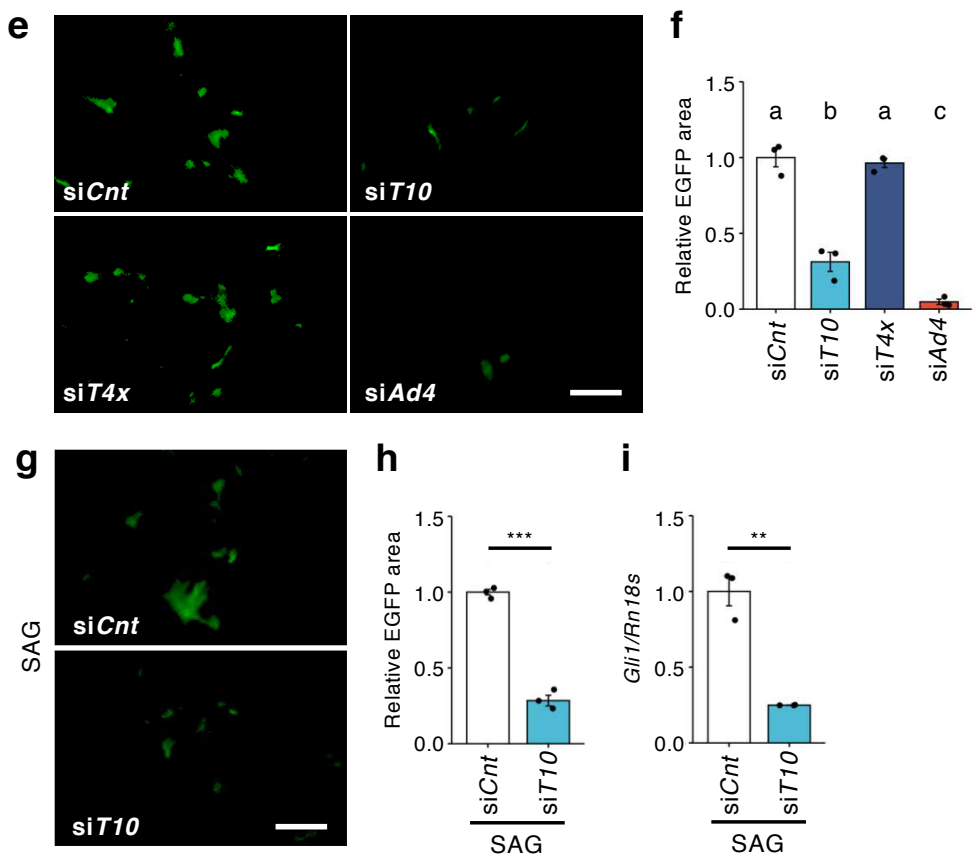

h

i

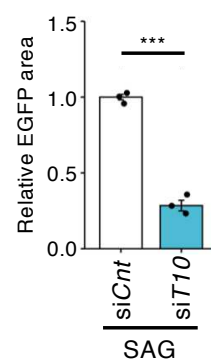

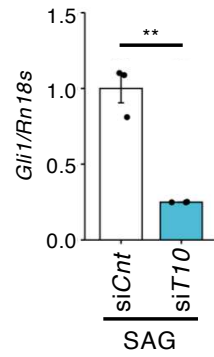




\section{Figure 3. Suppression of FLC differentiation by Tmsb10 KD}

(a) The experimental procedure for reconstruction of fetal testes. W-EGFP cells (E16.5) were plated and treated with siRNA for $24 \mathrm{~h}$. Whole testicular cells prepared from wildtype fetuses (E16.5) were mixed with siRNA-treated W-EGFP cells to reconstruct testes. The detailed procedure for testis reconstruction and culture is described in the Materials and Methods. (b) A representative image of the reconstructed testis. Testicular tubules (tu) formed, and FLCs with strong EGFP staining were observed in the interstitial regions of the reconstructed testes. (c) The reconstructed testes were cultured in the presence $(+)$ or absence (-) of SAG for 21 days. The reconstructed testes were examined under a fluorescence microscope to measure EGFP fluorescence. Scale bar $=100 \mu \mathrm{m}$. (d) The EGFP-positive cells (indicated by the relative EGFP-positive area) in the reconstructed testes above were analyzed quantitatively after incubation for 21 days. The cells were cultured in the absence (-, open bar) or presence of SAG (+, gray bar). $n=3 .{ }^{* * *} p<0.001$. (e) Representative images of the reconstructed testes using W-EGFP cells treated with the following siRNAs: control (siCnt), Tmsb10 (si T10), Tmsb4x (siT4x), and Ad4BP/SF-1 (siAd4). Scale bar $=100 \mu \mathrm{m}$. (f) The EGFP-positive cells in the reconstructed testes above were analyzed quantitatively. Letters $\mathrm{a}, \mathrm{b}$, and $\mathrm{c}$ denote significant differences between the cell groups treated with siCnt, siT10, siT4x, and siAd4. $n=3$. $p<0.001$. (g) Testes were reconstructed using W-EGFP cells treated with siCnt or siT10. They were cultured for 21 days in the presence of SAG. Scale bar $=100 \mu \mathrm{m}$. (h) The EGFP-positive cells in the reconstructed testes above were analyzed quantitatively. $n=3$. ${ }^{* * *} p<0.001$. (i) W-EGFP cells were treated with siCnt (open bar) or siT10 (light blue bar). Expression of Gli1 in the W-EGFP cells was examined by qRT-PCR. The data were normalized by Rn18s and are presented as means \pm SEM. $n=3 .{ }^{* *} p<0.01$. 

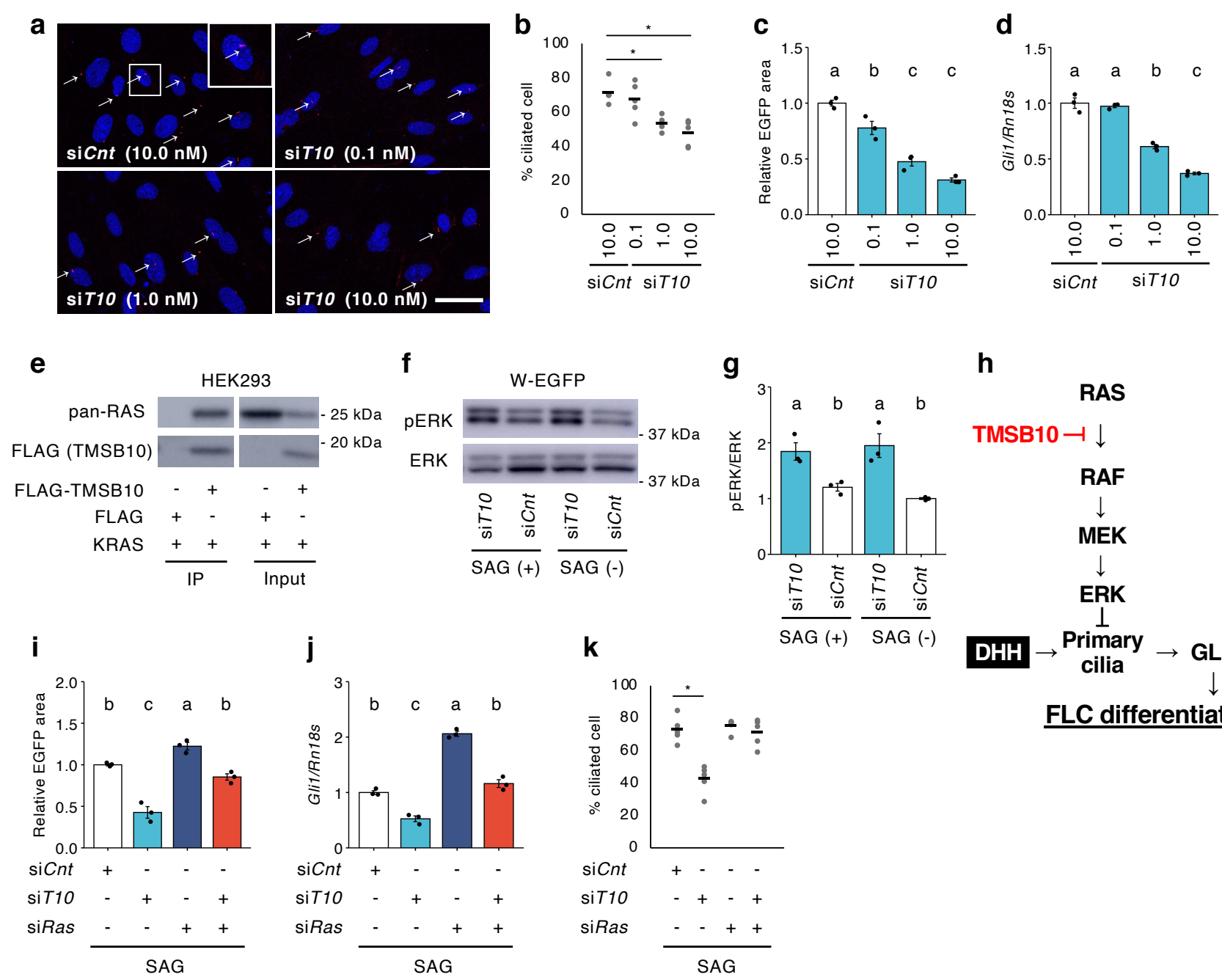

h

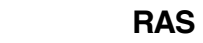

TMSB10 $\downarrow \downarrow$

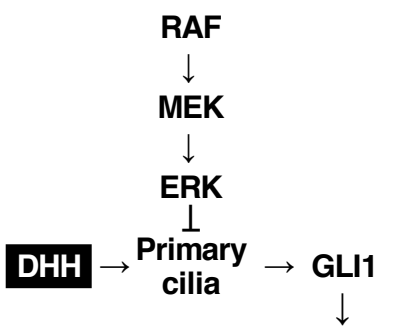

FLC differentiation 
Figure 4. Role of Tmsb10 in the regulation of ciliogenesis via suppression of the RAS/ERK pathway

(a) E16.5 W-EGFP cells were treated with $0.1,1.0$, or $10.0 \mathrm{nM}$ siT10 or $10.0 \mathrm{nM}$ siCnt, then cultured in serum-free medium for $24 \mathrm{~h}$. Thereafter, the cells were subjected to immunostaining for the cilial marker protein ARL13B (red). Nuclei were stained with 4',6'diamidino-2-phenylindole (DAPI) (blue). The enclosed area is enlarged at the top right. Arrows indicate primary cilia. Scale bar $=10 \mu \mathrm{m}$. (b) Ciliated cells detected in the studies above were counted. The ratios of the ciliated cells to all cells (\%) are shown. $n=5$. ${ }^{*} p<0.001$. (c) W-EGFP cells were treated with $0.1,1.0$, or $10.0 \mathrm{nM}$ siT10 or $10.0 \mathrm{nM}$ siCnt, then subjected to testis reconstruction assay. The EGFP-positive cells (indicated by the relative EGFP-positive area) in the reconstructed testes were analyzed quantitatively after incubation for 21 days. $n=3$. $p<0.001$. (d) W-EGFP cells were cultured and treated with $0.1,1.0$, or $10.0 \mathrm{nM}$ siT10 or $10.0 \mathrm{nM}$ siCnt. Expression of the Gli1 gene in the cells was examined. The data were standardized using Rn18s. $n=3$. $p<0.001$. (e) Interactions between TMSB10 and RAS were examined. Whole-cell lysates were prepared from HEK293 cells overexpressing FLAG-TMSB10 or FLAG together with KRAS. Proteins interacting with TMSB10 were immunoprecipitated with anti-FLAG antibody. The immunoprecipitates were subjected to immunoblotting using antibodies for pan-RAS and FLAG. The positions of molecular weight markers are indicated at the left. Full blot images are displayed in Supplemental Fig. 8. (f) Whole-cell extracts were prepared from E16.5 WEGFP cells treated with siT10 or siCnt in the presence (+) or absence (-) of SAG. Levels of phospho-ERK (pERK) and ERK were examined by Western blotting. (g) Signal intensities in the blots above were quantified as described in the Materials and Methods. The amounts of pERK relative to ERK are shown. $n=3$. $p<0.01$. (h) A schematic illustration summarizing the results so far. TMSB10 was assumed to suppress the RAS/ERK pathway by interacting with RAS. (i) W-EGFP cells were treated with siCnt (open bar), siT10 (light blue bar), siRas (dark blue bar), or both siT10 and siRas (red bar). These cells were then used for testis reconstruction. The reconstructed testes were cultured in the presence of SAG for 21 days. The EGFP-positive cells in the reconstructed testes were analyzed quantitatively. $n=3$. $p<0.001$. (j) W-EGFP cells were treated with siRNAs as above and then cultured in the presence of SAG. The expression of Gli1 was examined by qRT-PCR. The data were standardized using Rn18s. $n=3$. $p<0.001$. (k) WEGFP cells were treated with siRNAs as above, and their effect on ciliogenesis was examined by immunostaining for ARL13B. Ciliated cells were counted and the ratios of these cells to all cells (\%) are plotted. $n=5 .{ }^{*} p<0.001$. Letters a, b, and c on the bars in (c), $(d),(g),(i)$, and (j) denote significant differences between the cell groups. 

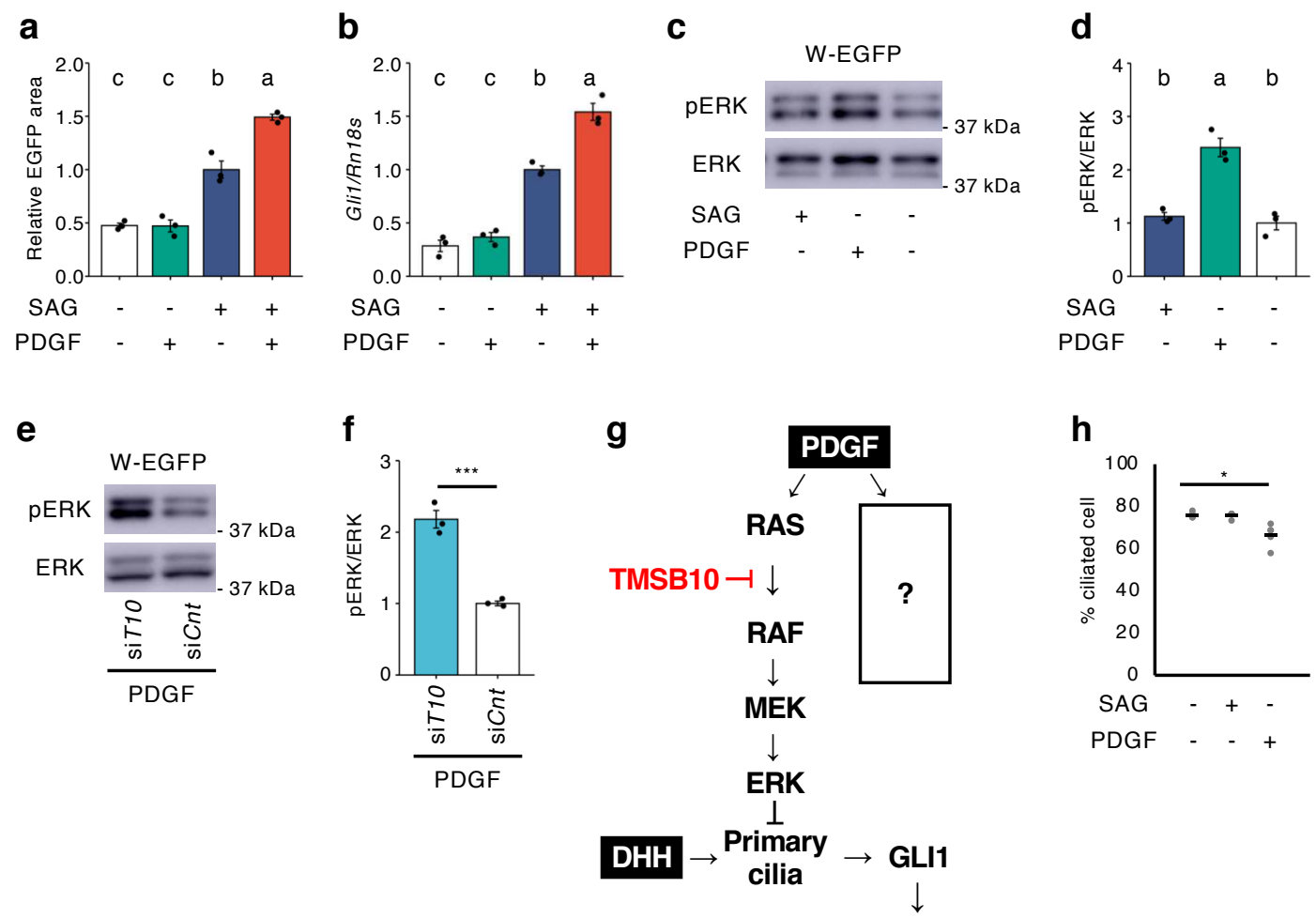

h

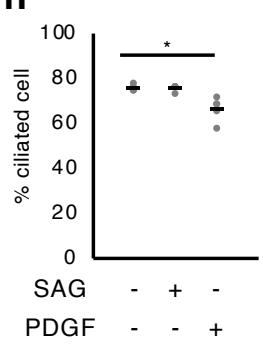

\section{FLC differentiation}




\section{Figure 5. Activation of the RAS/ERK pathway by PDGF}

(a) Reconstructed testes were incubated in the absence (open bar) or presence of PDGFAA (green bar), SAG (dark blue bar), or both PDGF-AA and SAG (red bar). The EGFPpositive cells in the reconstructed testes were analyzed quantitatively after incubation for 21 days. $n=3$. $p<0.001$. (b) W-EGFP cells were cultured under the same conditions as above. Expression of Gli1 in the cells was examined by qRT-PCR. The data were normalized by $R n 18 s$ and are presented as means \pm SEM. $n=3$. $p<0.01$. (c) Whole-cell extracts prepared from W-EGFP cells were cultured in the presence $(+)$ or absence (-) of PDGF-AA and subjected to Western blotting for ERK and pERK. The position of a molecular weight marker is indicated at the left. (d) Signal intensities of the blots above were quantified as described in the Materials and Methods. The amounts of $p E R K$ relative to ERK are shown. Letters a, b, and c on the bars in (a), (b), and (d) denote significant differences between the cell groups. $n=3$. $p<0.01$. (e) W-EGFP cells were treated with siRNA for siT10 and thereafter cultured in the presence (+) or absence (-) of PDGF-AA. Whole-cell extracts prepared from the cells were subjected to Western blotting for ERK and pERK. ( $f$ ) Signal intensities of the blots above were quantified. The amounts of $p E R K$ relative to ERK are shown. $n=3$. ${ }^{* *} p<0.001$. Full blot images for (c) and (d) are displayed in Supplemental Fig. 8. (g) A tentative schema for the function of PDGF is shown. It was assumed that another signal pathway could be activated downstream of PDGF. (h) WEGFP cells were cultured in the absence (-) or presence (+) of PDGF-AA and SAG. Ciliogenesis in the W-EGFP cells was examined by immunostaining for ARL13B. Ciliated cells were counted and the ratios of these cells to all cells (\%) are plotted. $n=3 .{ }^{*} p<0.001$. 
a

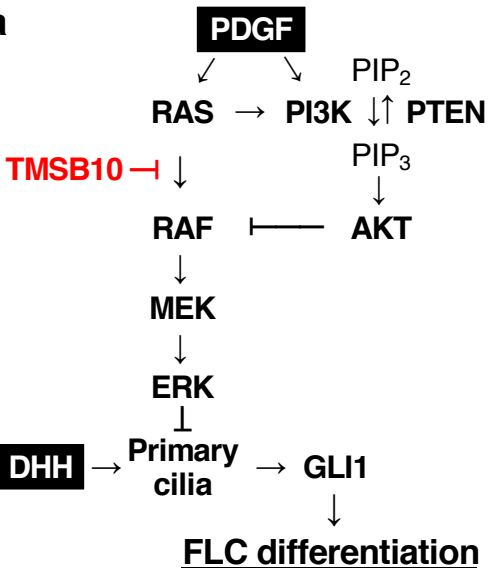

\section{d}
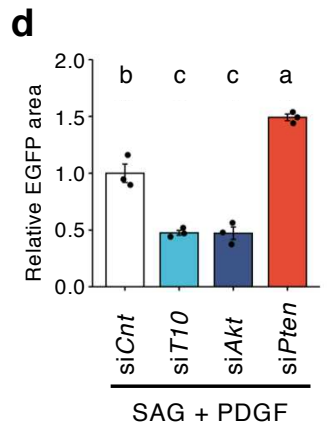

$\mathbf{g}$

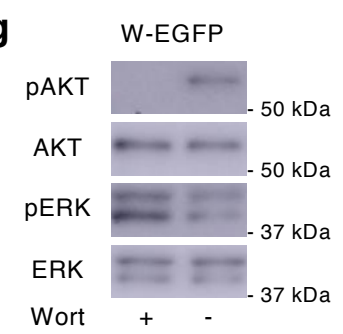

\section{i}

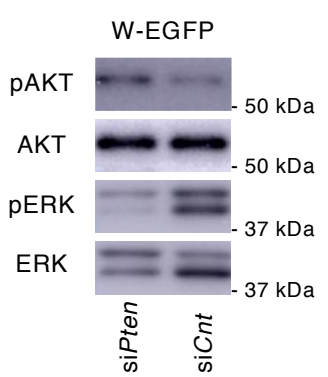

\section{e f}
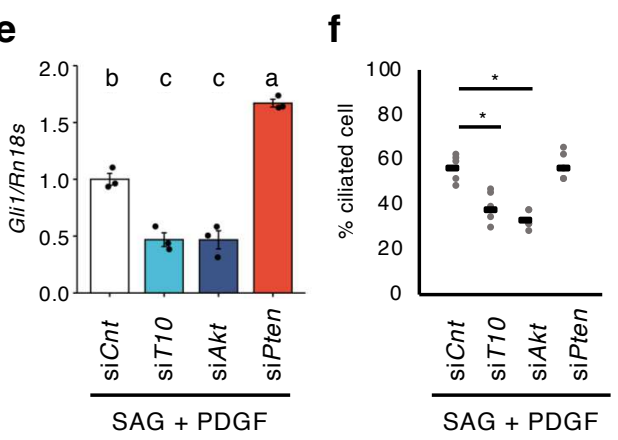

h

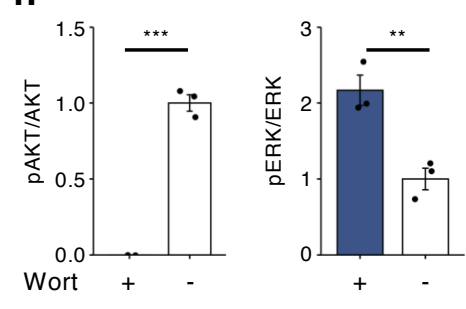

j

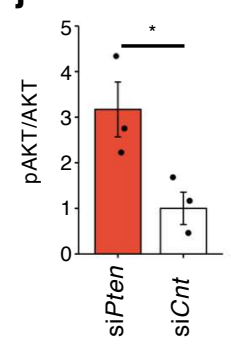

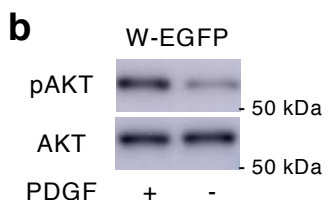

c

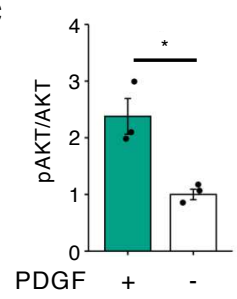




\section{Figure 6. Dual role of PDGF in regulating FLC differentiation}

(a) A schematic summary of signal pathways regulating FLC differentiation and in which Tmsb10 acts as a suppressor. (b) W-EGFP cells were cultured in the presence (+) or absence (-) of PDGF-AA. Whole-cell extracts prepared from the cells were subjected to Western blotting for phospho-AKT (pAKT) and AKT. Representative images of the blots are shown. The positions of molecular weight markers are indicated at the left. (c) Signal intensities in the blots above were quantified as described in the Materials and Methods. The amounts of pAKT relative to AKT are shown. $n=3$. ${ }^{*} p<0.05$. (d) W-EGFP cells were treated with siCnt, siT10, siAkt, or siPten, and then used for testis reconstruction. The reconstructed testes were cultured in the presence of SAG and PDGF for 21 days. The EGFP-positive cells in the reconstructed testes were quantified. $n=3$. $p<0.001$. (e) WEGFP cells were cultured under the same conditions as above. Expression of Gli1 in the cells was determined by qRT-PCR. The data were normalized by Rn18s and are presented as means \pm SEM. $n=3$. $p<0.001$. Letters $a, b$, and $c$ on the bars in $(d)$ and $(e)$ denote significant differences. ( $f$ ) W-EGFP cells were cultured under the same conditions as above. Ciliogenesis in the W-EGFP cells was examined by immunostaining for ARL13B. $n=5$. ${ }^{*} p<0.001$. (g) W-EGFP cells were cultured in the presence $(+)$ or absence (-) of wortmannin (Wort). Whole-cell extracts were subjected to Western blotting for pAKT, AKT, pERK, and ERK. (h) Signal intensities in the blots above were quantified as described in the Materials and Methods. The amounts of PAKT relative to AKT (left) and pERK relative to ERK (right) are shown. $n=3 .{ }^{* *} p<0.01 .{ }^{* * *} p<0.001$. (i) W-EGFP cells treated with siRNA for siPten and siCnt. Whole-cell extracts prepared from the cells were subjected to Western blotting for pAKT, AKT, pERK, and ERK. Full blot images for (b), (g), and (i) are shown in Supplemental Fig. 8. (j) Signal intensities in the blots above were quantified. The amounts of pAKT relative to AKT (left) and pERK relative to ERK (right) are shown. $n=3 .{ }^{*} p<0.05 .{ }^{* *} p<0.01$. 


\section{Supplementary Files}

This is a list of supplementary files associated with this preprint. Click to download.

- SuplnfoTmsb102022220214.pdf 